Aus dem Lehrbereich für Allgemeinmedizin der Albert-Ludwigs-Universität Freiburg i.Br.

\title{
Qualitätszirkel in der Sekundärprävention nach Schlaganfall - eine kontrollierte Interventionsstudie
}

\section{INAUGURAL-DISSERTATION}

\author{
zur \\ Erlangung des Medizinischen Doktorgrades \\ der Medizinischen Fakultät \\ der Albert-Ludwigs-Universität \\ Freiburg i.Br.
}

Vorgelegt: 2006

von Vera Mols

geboren in Bingen/Rh. 
1. Gutachter Prof. Dr. Wilhelm Niebling

2. Gutachter Prof. Dr. Andreas Hetzel Jahr der Promotion 2006 


\section{Inhaltsverzeichnis}

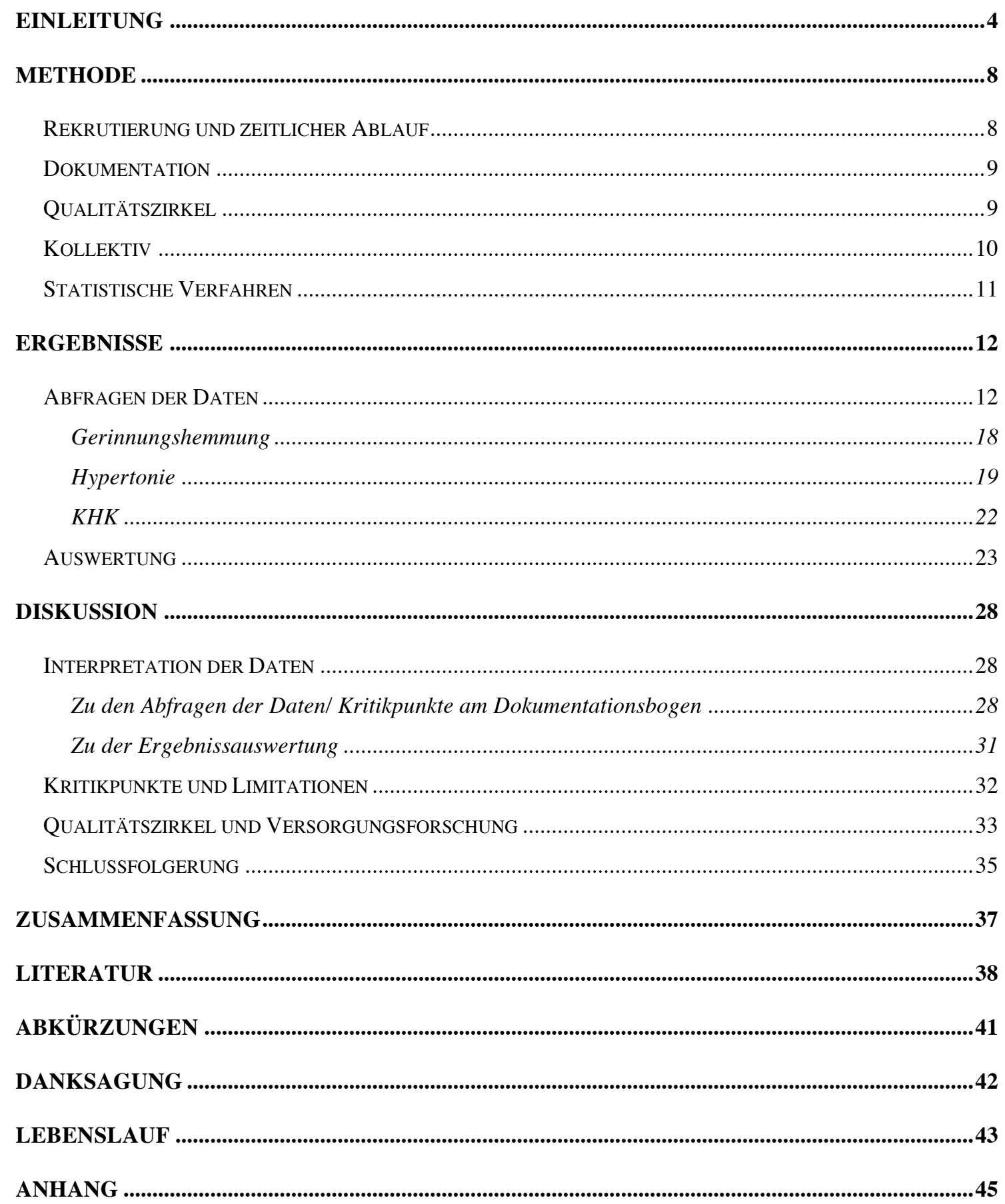




\section{Einleitung}

Der Schlaganfall ist eine akute, regional begrenzte Durchblutungsstörung des Gehirns, die durch intra- oder extrakranielle Gefäßstenosen bzw. -verschlüsse oder aber in selteneren Fällen durch Hirnblutungen zustande kommen kann. Die klinische Symptomatik ist charakterisiert durch reversible oder dauerhaft fokale neurologische Defizite wie Bewusstseinsstörungen, Lähmungen, sensomotorische Ausfälle, Aphasien oder Krampfanfälle. Nach der TOASTKlassifikation (Trial of Org 10172 in Acute Stroke Treatment) (1) unterscheidet man atherothrombotische, kardioembolische, mikroangiopathische Insulte und seltenere Ursachen wie Dissektionen, Gerinnungsanomalien und Blutungen. Die jährliche Inzidenz von Erstschlaganfällen bei über 45-jährigen liegt in der westlichen Welt bei ca. 400/100000 (7). Die Rehabilitation nach Schlaganfällen ist aufwändig (9), und viele Patienten haben keine Aussicht auf eine restitutio ad integrum (22). In den letzten Jahren wurde die Primärtherapie und Frührehabilitation nach Schlaganfall vor allem mit der breiten Einführung von sog. Stroke Units wesentlich verbessert $(8,25)$. Während die Primärprävention die Vermeidung von zerebralen Ischämien oder transitorischen ischämischen Attacken (TIAs) bei Patienten ohne bisherige zerebrovaskuläre Erkrankungen zum Ziel hat, gilt es bei der Sekundärprävention, nach Abschluss der Rehabilitation einen zweiten Schlaganfall zu verhindern. Der bedeutendste Risikofaktor für den Schlaganfall ist die arterielle Hypertonie (27). In den USA könnte man ca. ein Drittel aller zerebrovaskulären Ereignisse durch konsequente Blutdruckbehandlung verhindern (35). Neben nicht beeinflussbaren Risikofaktoren wie Alter, männlichem Geschlecht und genetischer Disposition spielen die beeinflussbaren Risikofaktoren Nikotinabusus, Diabetes mellitus, Übergewicht und Fettstoffwechselerkrankungen sowie Vorhofflimmern, Herzklappenfehler und Carotisstenose die wichtigste Rolle.

Die Patienten werden nach Erstschlaganfall aus dem Krankenhaus und der Rehabilitationseinrichtung mit dezidierten Therapieempfehlungen in die hausärztliche Versorgung entlassen. Da medizinische Erkenntnisse im Allgemeinen 
nur ca. zur Hälfte in die Praxis umgesetzt werden (30), ist es wahrscheinlich, dass es auch bei der Versorgung von Patienten mit Schlaganfall an der Umsetzung von medizinischen wissenschaftlichen Empfehlungen in die Praxis mangelt. Als Antwort auf diese Problematik wurden bereits in den 90er Jahren in Deutschland Leitlinien entwickelt, die eine Verbesserung der Versorgungsqualität zum Ziel hatten. Die Bundesärztekammer (BÄK) und die Kassenärztliche Bundesvereinigung (KBV) definierten im Jahr 1997 in Anlehnung an die Definition von Field und Lohr aus dem Jahr 1990 (13) Leitlinien als „systematisch entwickelte Entscheidungshilfen über die angemessene ärztliche Vorgehensweise bei speziellen gesundheitlichen Problemen“ (5). Leitlinien geben dabei einen Handlungsspielraum vor, von dem nur im begründeten Einzelfall abgewichen werden kann. Allerdings sind Leitlinien insofern problematisch, da auch sie schwierig umzusetzen sind. Es kam zu einer schnell anwachsenden Anzahl von Leitlinien mit teilweise widersprüchlichen Handlungsempfehlungen und mangelnder Abstimmung untereinander. Darüber hinaus existieren häufig weitere wichtige Qualitätsdefizite der einschlägigen Leitlinien (26):

1. Fehlende bzw. unzureichende Dokumentation der zugrunde liegenden Evidenz bzw. der Auswahlkriterien für bestimmte Empfehlungen.

2. Unzureichende Dokumentation der Autoren bzw. der Verfahren zur Konsensbildung.

3. Fehlende Kosten-Nutzen-Kalkulation.

Aufgrund der erwähnten qualitativen Defizite vieler Leitlinien ist es nicht erstaunlich, dass sie nur einen teils geringen Umsetzungsgrad haben. Besonders fehlende bzw. unzureichende Evidenz einer Leitlinie wirkt sich ungünstig auf ihre Umsetzung aus (17).

Zwischen 1999 und 2004 wurde von der Ärztlichen Zentralstelle für Qualitätssicherung ein Clearingverfahren für Leitlinien eingerichtet. So existiert seit dem 26.01.2005 auch ein Clearingbericht zum Thema Sekundärprävention nach Schlaganfall (10). Inwieweit dies nun zur Verbesserung der Implementierung beitragen wird, ist noch nicht bekannt. 
Vor diesem Hintergrund versucht man seit einigen Jahren, die Umsetzung bestimmter Maßnahmen durch Qualitätszirkelarbeit zu steigern. In den letzten zehn Jahren ist die Anzahl von durch die kassenärztl. Vereinigung anerkannten Qualitätszirkel von anfangs 15 auf 4900 Qualitätszirkel im Jahr 2004 gestiegen (20). Während es einige Evaluationen bezüglich der Ziele von Qualitätszirkeln gibt, existieren wenige Arbeiten über ihre Ergebnisqualität (20). Qualitätszirkel können ein Baustein in einer Vielzahl von Projekten sein, die den Umsetzungsgrad der Leitlinien in die Praxis erhöhen (26). Sie verbessern den Wissensstand der Teilnehmer, und sie geben Gelegenheit zur Reflexion der täglichen Arbeit. Eine Interaktionsanalyse im Jahr 2000 zeigte jedoch, dass eine deutliche Diskrepanz zwischen Zielen und Anforderungen an Qualitätszirkel und ihrer konkreten Umsetzung in der Praxis besteht (33). Hierbei wurde deutlich, dass die Qualitätszirkelarbeit als zu beliebig verstanden wird und selten zur Erarbeitung verbindlicher Leitlinien genutzt wird. Trotzdem haben sich Qualitätszirkel in verschiedenen Bereichen als sinnvoll erwiesen $(14,34)$.

Die vorliegende Arbeit will die Frage beantworten, ob Qualitätszirkel zur Verbesserung der hausärztlichen Versorgungsqualität beitragen. Das Projekt wurde vom Kompetenznetz Schlaganfall ins Leben gerufen. Dieser bundesweite Verbund klinisch-wissenschaftlicher Einrichtungen, Organisationen und Selbsthilfegruppen befasst sich mit der Pathophysiologie, Diagnostik, Therapie und Prävention des Schlaganfalls. Die Frage nach der Umsetzung diesbezüglicher Erkenntnisse in die klinische Praxis ist Gegenstand der Versorgungsforschung, welche ein neuer wichtiger wissenschaftlicher Schwerpunkt des Kompetenznetzes ist. Das hier dargestellte Projekt steht am Beginn einer Reihe von derartigen Untersuchungen. Die Leitung übernahmen gemeinsam die Klinik für Neurologie und der Lehrbereich für Allgemeinmedizin der Universität Freiburg. Finanziell wurde die Studie vom Bundesministerium für Bildung und Forschung unterstützt. Nach einer PowerAnalyse wurde geschätzt, dass etwa 400 Patienten untersucht werden müssen, um einen positiven Effekt von Qualitätszirkeln auf die Blutdruck- und Cholesterinwerteinstellung, Antikoagulation und antithrombotische Behandlung mit einer 80-prozentigen Wahrscheinlichkeit auf dem Signifikanzniveau 0,05 
belegen zu können. Um diese relativ große Patientenzahl rekrutieren zu können, sollte das Projekt ursprünglich gemeinsam mit der Heidelberger Universitätsklinik durchgeführt werden. Aus organisatorischen Gründen war die angestrebte Kooperation jedoch nicht möglich. Daher musste davon Abstand genommen werden, die Ergebnisqualität der Qualitätszirkelarbeit an Hand der vier erwähnten Parameter zu untersuchen. Das verkleinerte Projekt konzentrierte sich nun auf die Struktur- und Prozessqualität, wobei an den vier Risikofaktoren festgehalten wurde. Dazu wurde ein Qualitätsindikatorenset verwendet, welches zuvor von der Freiburger Arbeitsgruppe (24) erstellt worden war. Anschließend wurde ein Dokumentationsbogen entwickelt, an Hand dessen Patientendaten aus Hausarztpraxen erhoben wurden. Da der Effekt quantitativ nicht abschätzbar war, gab es keine Grundlage für eine erneute Power-Analyse. Damit hat die Untersuchung den Charakter einer Pilotstudie.

Bei der vorgelegten Untersuchung handelt es sich um eine prospektive, kontrollierte Interventionsstudie, in der untersucht wurde, ob sich die Fortbildung der Hausärzte durch Qualitätszirkel (QZ) in einer messbaren Verbesserung der Betreuung von Schlaganfallpatienten niederschlägt. 


\section{Methode}

Es wurden Patientendaten aus Hausarztpraxen im südlichen Schwarzwald erhoben. In drei Orten wurden Qualitätszirkel angeboten, deren teilnehmende Ärzte aus 20 Ortschaften die Interventionsgruppe bildeten. Hausärzte aus 13 weiteren Orten bildeten sich auf traditionelle Art weiter und formierten die Kontrollgruppe.

\section{Rekrutierung und zeitlicher Ablauf}

Patienten nach cerebralem ischämischen Ereignis wurden im Zeitraum vom 15.11.2000 bis 31.3.2002 aus vier Akutkrankenhäusern (Furtwangen, TitiseeNeustadt, Donaueschingen, Freiburg) befragt, ob sie an der Studie teilnehmen möchten. Eine schriftliche Einverständniserklärung der Patienten bzw. ihrer Angehörigen und ein positives Votum der Ethikkommission lagen vor. Anschließend wurden die betreuenden Hausärzte, die in maximaler Distanz von $20 \mathrm{~km}$ zu der primärversorgenden Klinik niedergelassen waren, gebeten, an der Studie teilzunehmen. Patienten, deren Hausärzte die Teilnahme ablehnten, wurden aus der Studie ausgeschlossen. In Kirchzarten, Furtwangen und Titisee-Neustadt wurden von März 2002 bis März 2003 Qualitätszirkel angeboten. Die dort und im nahen Umkreis praktizierenden Ärzte wurden gefragt, ob sie am Qualitätszirkel teilnehmen möchten. Sie bildeten die Interventionsgruppe. Die Kontrollgruppe bestand aus der Ärzteschaft der übrigen Orte und fünf Ärzten, die zwar im Interventionsgebiet arbeiteten, aber nicht an den Qualitätszirkeln teilnehmen wollten.

6 Monate nach Durchführung der Qualitätszirkelarbeit wurde begonnen, die Behandlungsdaten aus den einzelnen Arztpraxen im Zeitraum vom September 2003 bis zum September 2004 zu dokumentieren. 


\section{Dokumentation}

Mit der Entwicklung und Zusammenstellung der für die Fragestellung benötigten Qualitätsindikatoren durch die Arbeitsgruppe des Lehrbereichs für Allgemeinmedizin und der Neurologischen Klinik in Freiburg (14) war die Grundlage für die Studie geschaffen. Es wurde anschließend der Dokumentationsbogen (s. Anhang) entwickelt, anhand dessen die Behandlungsdaten erhoben wurden. Vier wissenschaftliche Hilfskräfte, zu denen auch die Autorin dieses Textes gehörte, waren mit dieser Aufgabe beschäftigt. Mit einem von der Klinik zur Verfügung gestellten PKW wurden die Praxen im Schwarzwald angefahren und der Dokumentationsbogen für jeden Patienten vor Ort ausgefüllt. Die notwendigen Daten wurden aus der Patientenakte bzw. der elektronischen Dokumentation entnommen. Der Zeitaufwand betrug im Durchschnitt eine Stunde pro Patient. Die Daten wurden anschließend in eine elektronische Datenbank (Microsoft Access für Windows) übertragen.

Relevante Qualitätsindikatoren für die Sekundärprävention nach Schlaganfall beziehen sich auf folgende hausärztlich beeinflussbare Variablen: Diagnostik und Therapie der arteriellen Hypertonie, antithrombotische Behandlung, Antikoagulation bei Vorhofflimmern, Nikotinabusus und Hypercholesterinämie. Das Indikatorenset für Hypertonie, Nikotinabusus und Hypercholesterinämie wurde nach den Empfehlungen der Ärztlichen Zentralstelle für Qualitätssicherung in der Medizin (ÄZQ) und aus dem von Marshall et al. publizierten Indikatorenset ausgewählt $(2,29)$. Die Qualitätsindikatoren zur antithrombotischen Behandlung und Antikoagulation wurden anhand einer Übersichtsarbeit von Hetzel entwickelt (23).

\section{Qualitätszirkel}

Die Qualitätszirkelsitzungen fanden im 6 - 8 wöchentlichen Rhythmus statt und wurden von einem durch die Akademie für ärztliche Fort- und Weiterbildung Südbaden geschulten Moderator (Herr H. Jahn, Lehrbereich Allgemeinmedizin Freiburg) geleitet. Vorträge eines Neurologen über das 
Akutbehandlungskonzept, neurologische Messverfahren (Barthel-Index (28), Modifizierte Rankin Skala (MRS) $(3,31)$, National Institutes of Health Stroke Scale (NIH-SS) $(3,6))$ und die antithrombotische Behandlung nach Schlaganfall führten in das Thema ein. Die Qualitätszirkelarbeit orientierte sich inhaltlich an der Beeinflussung der Risikofaktoren für Schlaganfall (Hypertonieeinstellung, antithrombotische Behandlung, Antikoagulation, lipidsenkende Maßnahmen und praktische Umsetzung der therapeutischen Ziele in der Hausarztpraxis). Weitere Themen waren Evidence-based Medicine (EBM) und Literaturrecherche im relevanten Kontext.

Die Anzahl der Teilnehmer pro Qualitätszirkel lag zwischen 8 und 20 Ärzten. Die kleinste Arbeitsgruppe in Kirchzarten bestand schon vor Beginn der Studie und hatte acht Teilnehmer, die sich an neun Terminen trafen. Der Qualitätszirkel Furtwangen bestand aus 17 Hausärzten, die sich an sechs Terminen trafen. Der größte Zirkel fand mit 20 Teilnehmern in Titisee-Neustadt statt. Die Teilnehmer trafen sich an sieben Terminen. Die unterschiedliche Anzahl von Sitzungen hatte organisatorische Gründe. Inhaltlich wurden in allen drei Zirkeln die gleichen Themen behandelt. Einzig in Titisee-Neustadt kam es nicht mehr zur Bearbeitung des Risikofaktors Hyperlipidämie. Die Beteiligung war weitgehend regelmäßig. Bei den wenigen Ärzten, die nicht alle Termine wahrnehmen konnten, nahmen wir trotzdem einen Interventionseffekt an.

\section{Kollektiv}

Insgesamt wurden 212 Patienten für die Studie rekrutiert. Eingeschlossen wurden Patienten mit einer transitorischen ischämischen Attacke (TIA), einem prolongierten reversiblen ischämischen neurologischen Defizit (PRIND) oder einem Schlaganfall mit NIH-SS $<20(3,6), \operatorname{MRS}<5(3,31)$ und Barthel-Index $>30$ (28). Wie aus Abb. 1 hervorgeht, konnten die Daten von 126 Patienten ausgewertet werden, die von 61 Hausärzten versorgt wurden (55 Einzel- und sechs Gemeinschaftspraxen). Die Ärzte behandelten zwischen mindestens einem bis maximal sieben Patienten. 


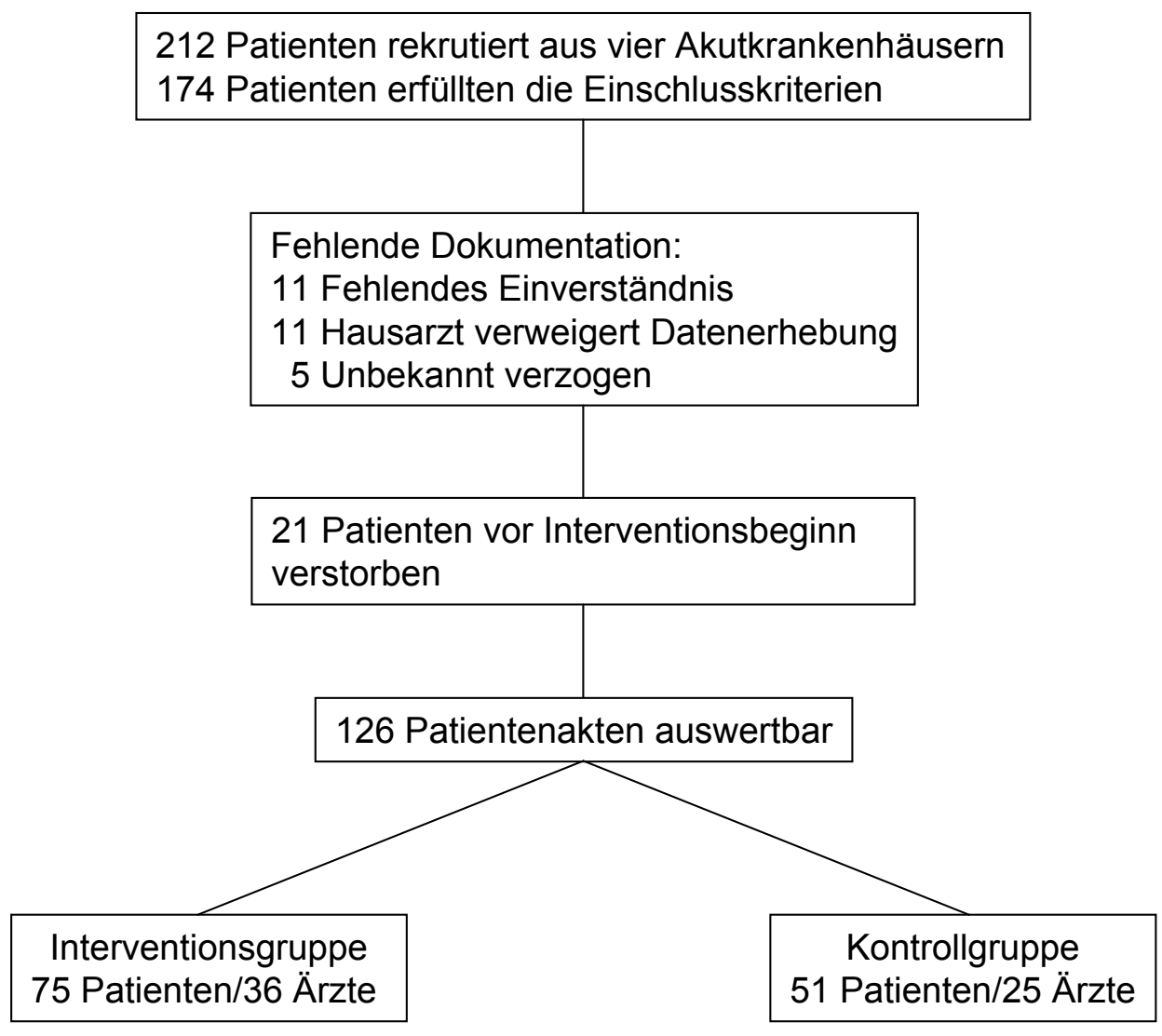

Abb. 1: Rekrutierung und Ausschluss der Patienten. Zuteilung zu Interventions- und Kontrollgruppe

\section{Statistische Verfahren}

Nach einer statistischen Beratung durch Herrn Dipl.-Stat. M. Olschewski (Universität Freiburg, Medizinische Biometrie und Statistik) wurden zum Vergleich der Behandlungsparameter zwischen Interventionsgruppe und Kontrollgruppe der Vierfelder-Test nach Fisher und der t-Test für ungepaarte Stichproben verwendet (StatView 5.0.1 für Apple Macintosh), der McNemar-Test wurde manuell errechnet (32). Zwei Items wurden mit Hilfe von Herrn Dr. M. Nübling (Gesellschaft für Empirische Beratung, Denzlingen) mit einem multiplen Mittelwertvergleich (LSD: Least Significant Difference) evaluiert. 


\section{Ergebnisse}

\section{Abfragen der Daten}

Die Daten wurden vom Dokumentationsbogen in eine Microsoft-Access-Maske eingegeben. Diese beinhaltete den Dokumentationsbogen in elektronischer Form sowie eine tabellarische Übersicht der Daten. Mit Hilfe von Herrn Florian Weitling (Informatiker, Universität Freiburg) wurden die Daten von Access in Microsoft Excel eingelesen, bearbeitet und in My SQL 4.1 unter Windows übertragen. Nun wurden die Qualitätsindikatoren anhand von Abfragen der Daten untersucht. Die Vorgehensweise wird im Folgenden erläutert. Bei den Zahlenangaben sind Interventions- und Kontrollgruppe zusammengefasst. Die Aufspaltung der beiden Gruppen ist aus Tabelle $1 \mathrm{zu}$ ersehen. 


\section{Datenabfragen}

\section{Antithrombotische Behandlung/Antikoagulation}

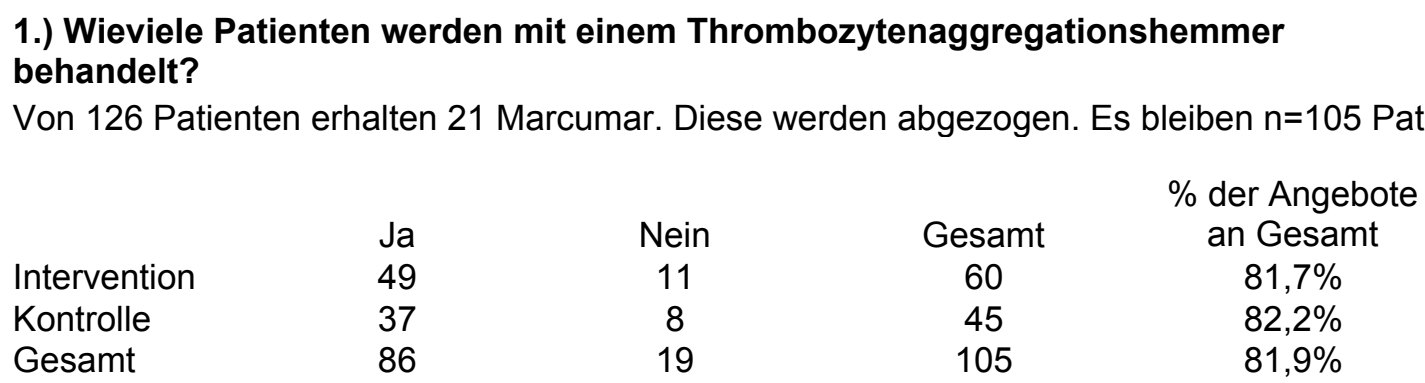

Wieviele Patienten haben Marcumar bekommen?

$\begin{array}{lccc} & \text { Ja } & \text { Gesamt } & \% \text { an Gesamt } \\ \text { Intervention } & 15 & 75 & 20,0 \% \\ \text { Kontrolle } & 6 & 51 & 11,8 \% \\ \text { Gesamt } & 21 & 126 & 16,7 \%\end{array}$

2.) Wievielen Patienten, die die Diagnose Vorhofflimmern haben oder eine kardioembolische Genese des Schlaganfalls (TOAST b.), wird Marcumar verordnet?

$\begin{array}{lccc} & \text { VHF, TOAST b } & \text { Marcumar ja } & \% \text { an Gesamt } \\ \text { Intervention } & 10 & 10 & 100,0 \% \\ \text { Kontrolle } & 1 & 1 & 100,0 \% \\ \text { Gesamt } & 11 & 11 & 100,0 \%\end{array}$

Hier dargestellt ist die Anzahl der Tests.

\begin{tabular}{|c|c|c|c|c|}
\hline & & $\begin{array}{l}\text { Quick-Teste } \\
\text { [20\%-33\% }\end{array}$ & & \\
\hline & Quick-Teste & einschl.] & INR & INR [2-3] \\
\hline Intervention & 48 & 23 & 662 & 424 \\
\hline Kontrolle & 17 & 11 & 177 & 72 \\
\hline Gesamt & 65 & 34 & 839 & 496 \\
\hline & vor In & tion & & \\
\hline & INR & INR [2-3] & INR & INR [2-3] \\
\hline Intervention & 116 & 69 & 326 & 222 \\
\hline Kontrolle & 16 & 7 & 85 & 30 \\
\hline Gesamt & 132 & 76 & 411 & 252 \\
\hline
\end{tabular}

Die Quickwerte werden nicht gezählt, wenn ein gültiger INR vorhanden ist. Zeiträume: vor I.: 01.03.2000 - 31.08.2001; nach I.: 01.03.2002 - 31.08.2003 (je 18 gleiche Monate) 


\section{Risikofaktor Hypertonie}

\begin{tabular}{|c|c|c|c|}
\hline Wieviele Pa & ten haben die & gnose "Hypert & \\
\hline & Diagnosenliste & Diagnosenliste & \\
\hline & mit Datum & ohne Datum & Item H_2 \\
\hline Intervention & 17 & 45 & 52 \\
\hline Kontrolle & 24 & 44 & 44 \\
\hline Gesamt & 41 & 89 & 96 \\
\hline
\end{tabular}

4.) Ist die Diagnose Hypertonie in der Praxisdokumentation klar erkennbar? n=96 Patienten mit Diagnose Hypertonie aus Item H_2. Klare Dokumentation ist ein Eintrag in der Diagnosenliste.

$\begin{array}{lccc} & \text { Ja } & \text { Gesamt } & \% \text { an Gesamt } \\ \text { Intervention } & 45 & 52 & 86,5 \% \\ \text { Kontrolle } & 44 & 44 & 100,0 \% \\ \text { Gesamt } & 89 & 96 & 92,7 \%\end{array}$

5.)Wurde bei einem Blutdruckwert $>=160 / 100 \mathrm{mmHg}$ innerhalb von drei Monaten eine Kontrollmessung durchgeführt?

\begin{tabular}{|c|c|c|c|c|}
\hline & \multicolumn{4}{|c|}{\begin{tabular}{|l|l|l|l} 
mind. eine Wiederholungsmessung keine Wiederholungsmessung \\
\end{tabular}} \\
\hline & vor Intervention & bei/nach Interv. & vor Intervention & bei/nach Interv \\
\hline Intervention & 309 & 68 & 86 & 41 \\
\hline Kontrolle & 232 & 61 & 57 & 19 \\
\hline Gesamt & 541 & 129 & 143 & 60 \\
\hline & $>=160 / 100$ ges. & & & \\
\hline Intervention & 504 & & & \\
\hline Kontrolle & 369 & & & \\
\hline Gesamt & 873 & & & \\
\hline
\end{tabular}

Hier wurde die Anzahl der Blutdruckmessungen mit RR >= 160/100 gezählt, die keine/mind. eine Wiederholungsmessung innerhalb von 3 Monaten hatten. Besonderheit: Die erste Messung kann bis zu 3 Monate vor Interventionsbeginn liegen, wenn die zweite Messung nach I.beginn ist bzw. bis nach I.beginn keine zweite Messung stattgefunden hat.

6.) Wurden innerhalb von 3 Monaten nach oder vor der Erstdiagnose der Hypertonie folgende Erkrankungen und Risikofaktoren überprüft, diagnostiziert bzw. ausgeschlossen? $\mathrm{n}=41$ Patienten mit Diagnosendatum

$\begin{array}{lccc}P A V K & \text { Überprüft } & \text { Hypertoniker } & \% \text { an Hypertonikern } \\ \text { Intervention } & 1 & 17 & 5,9 \% \\ \text { Kontrolle } & 2 & 24 & 8,3 \% \\ \text { Gesamt } & 3 & 41 & 7,3 \% \\ & & & \\ \text { Diabetiker } & \text { Überprüft } & \text { Hypertoniker } & \% \text { an Hypertonikern } \\ \text { Intervention } & 1 & 17 & 5,9 \% \\ \text { Kontrolle } & 3 & 24 & 12,5 \% \\ \text { Gesamt } & 4 & 41 & 9,8 \%\end{array}$




$\begin{array}{lccc}\text { Hyperlipidämie } & \text { Überprüft } & \text { Hypertoniker } & \% \text { an Hypertonikern } \\ \text { Intervention } & 1 & 17 & 5,9 \% \\ \text { Kontrolle } & 2 & 24 & 8,3 \% \\ \text { Gesamt } & 3 & 41 & 7,3 \% \\ & & & \\ \text { Nikotinabusus } & \text { Überprüft } & \text { Hypertoniker } & \% \text { an Hypertonikern } \\ \text { Intervention } & 0 & 17 & 0,0 \% \\ \text { Kontrolle } & 0 & 24 & 0,0 \% \\ \text { Gesamt } & 0 & 41 & 0,0 \% \\ & & & \\ \text { Alkoholkonsum } & \text { Überprüft } & \text { Hypertoniker } & \% \text { an Hypertonikern } \\ \text { Intervention } & 0 & 17 & 0,0 \% \\ \text { Kontrolle } & 0 & 24 & 0,0 \% \\ \text { Gesamt } & 0 & 41 & 0,0 \%\end{array}$

7.) Wieviel Patienten mit Zielorganschaden bekommen bei $3 x$ RR $>=140 / 100 \mathrm{mmHg}$ in Folge eine medikamentöse Therapie verordnet?

$\mathrm{n}=12$ Patienten, die nach Interventionsbeginn $3 x$ Werte $>=140 / 90 \mathrm{mmHg}$ in Folge haben.

Intervention

Kontrolle

Gesamt

\begin{tabular}{|c|c|c|c|}
\hline \multicolumn{2}{|c|}{$3 \times R R>=140 / 90$ in Folge } & $\begin{array}{c}3 \times R R>=140 / 90 \text { in Folge \& mind.eine } \\
\text { Medikation }\end{array}$ \\
\hline vor Intervention & nach Interv. & vor Intervention & nach Interv. \\
\hline
\end{tabular}

113

$35 \quad 12$

$\begin{array}{ll}9 & 19 \\ 3 & 10\end{array}$

29
2

10

\section{8.) Wievielen Patienten mit diagn. Hypertonie wurden die folgenden nicht- medikamentösen Massnahmen empfohlen? \\ $\mathrm{n}=8$ Patienten mit Hypertonie und $\mathrm{BMl}>30$ \\ Gewichts- \\ reduktion bei

$$
\text { Hypertoniker }
$$$$
\text { (H_2) }
$$ \\ Intervention \\ Kontrolle \\ Gesamt \\ $\mathrm{BMI}>30$ \& Hypertoniker

44 \\ 96

5
3
8

Empfohlen
4
3
7 \\ \%Empf./BMI>30 \\ \&Hypertonie \\ $80,0 \%$ \\ $100,0 \%$ \\ $87,5 \%$}

n=12 Patienten mit Hypertonie und schädlichem Alkoholkonsum

Begrenzung

des Alkohol-

konsums

Intervention

Kontrolle

Gesamt

$\begin{array}{cc}\begin{array}{c}\text { Hypertoniker } \\ \left(\mathrm{H}_{2} 2\right)\end{array} & \begin{array}{c}\text { Alkoholkonsum } \\ \text { \& Hypertonie }\end{array} \\ 52 & 6 \\ 44 & 6 \\ 96 & 12\end{array}$

\% Empf. I

Alkoholkonsum

\& Hypertonie

$33,3 \%$

$33,3 \%$

$33,3 \%$ 
9.) Erhalten Patienten unter antihypertensiver Medikation mind. 1x pro Jahr eine RRMessung?

$\mathrm{n}=77$ Patienten erhalten eine antihypertensive Medikation. Diese Gruppe teilt sich ohne Rest auf in "mit" und "ohne" jährliche Blutdruckmessung.

\begin{tabular}{|c|c|c|c|}
\hline \multirow[b]{3}{*}{ Intervention } & \multicolumn{3}{|c|}{ antihypertensive Medikation\&mind.1xRR/a } \\
\hline & vor Intervention & nach Interv. & Gesamtzeitraum \\
\hline & 25 & 42 & 24 \\
\hline Kontrolle & 21 & 23 & 15 \\
\hline \multirow[t]{3}{*}{ Gesamt } & 46 & 65 & 39 \\
\hline & antihypertens & Medikation8 & t mind. 1xRR/a \\
\hline & vor Intervention & nach Interv. & Gesamtzeitraum \\
\hline Intervention & 21 & 4 & 22 \\
\hline Kontrolle & 10 & 8 & 16 \\
\hline Gesamt & 31 & 12 & 38 \\
\hline
\end{tabular}

10.) Bekommen Patienten mit RR>150/90mmHg 3x in Folge eine Therapieänderung?

\begin{tabular}{lcc|c|c|} 
& $\begin{array}{c}3 \times \mathrm{RR}>150 / 90 \\
\text { über RR-Tab. }\end{array}$ & $\begin{array}{c}\text { 3x RR }>150 / 90 \\
\text { in Folge über RR } \\
\text { Tab. }\end{array}$ & $\begin{array}{c}\text { mind. } 3 x>150 / 90 \\
\text { über H_12a }\end{array}$ \\
\cline { 2 - 4 } Intervention & 48 & 15 & 28 \\
Kontrolle & 31 & 4 & 21 \\
Gesamt & 79 & 19 & 49
\end{tabular}

Die 1. u. 2. Spalte sind über die Blutdrucktabelle ermittelt. $H_{-}$12a fordert keine Werte in Folge. Es muss die 2. Spalte verwendet werden.

11.) Wurden Nierenwerte im Zeitraum von 6 Monaten, bevor die Behandlung mit einem ACE-Hemmer begann und im Zeitraum von 1 Monat nach Behandlungsbeginn überprüft?

$\begin{array}{lcc} & \begin{array}{c}\text { nur erster ACE- } \\ \text { Hemmer }+ \\ \text { Überprüfung }\end{array} & \begin{array}{c}\text { ACE-Hemmer- } \\ \text { Gabe gesamt }\end{array} \\ \text { Intervention } & 6 & 43 \\ \text { Kontrolle } & 2 & 25 \\ \text { Gesamt } & 8 & 68\end{array}$

\section{Nikotinabusus}

12.) Wurde bei Nikotinabusus die Empfehlung zur Nikotinkarenz gegeben?

Intervention

Kontrolle

Gesamt

$\begin{array}{cc}\text { Nikotin } & \text { \&Empfehlung } \\ 10 & 3 \\ 9 & 3 \\ 19 & 6\end{array}$




\section{KHK}

13.) Ist die Diagnose KHK in der Praxisdokumentation klar erkennbar?

$\begin{array}{lcc} & \text { KHK } & \text { KHK in } \\ \text { Diagn.liste }\end{array}$

14.) Ist das Serumcholesterin innerhalb der letzten 5 Jahre gemessen worden?

$\begin{array}{lcc}\text { alle Pat. mit } & \begin{array}{c}\text { KHK und } \\ \text { Cholesterin- } \\ \text { kontrolle }\end{array} & \begin{array}{c}\text { Cholesterin- } \\ \text { kontrolle }\end{array} \\ \text { Intervention } & 62 & 25 \\ \text { Kontrolle } & 33 & 7 \\ \text { Gesamt } & 95 & 32\end{array}$

\section{KHK und Cholesterin $>200$ mg/dl}

15.) Ist bei diesen Patienten eine Ernährungsberatung durchgeführt worden? Ernährungsberatung Intervention Kontrolle vor Intervention nach Intervention

Gesamt

$\begin{array}{ll}4 & 1 \\ 1 & 0 \\ 5 & 1\end{array}$

16.) Bekamen diese Patienten eine medikamentöse Therapie? med. lipidsenk.

Th.

Intervention

Kontrolle

Gesamt vor Intervention nach Intervention

$\begin{array}{ll}6 & 3 \\ 1 & 1 \\ 7 & 4\end{array}$

17.) Bekamen diese Patienten eine Therapieänderung?

Therapie-

änderung vor Intervention nach Intervention

Intervention $\quad 0 \quad 3$

Kontrolle $\quad 0 \quad 0$

Gesamt $\quad 0 \quad 3$ 


\section{Komorbiditäten}

\begin{tabular}{|c|c|c|c|c|}
\hline & $\mathrm{BMI}>=30\left(\mathrm{H} \_9 \mathrm{e}\right)$ & $\begin{array}{c}\text { Diabetiker } \\
\text { (H_5b1) }\end{array}$ & $\begin{array}{l}\text { Diabetiker über } \\
\text { Diagnosenliste }\end{array}$ & $\begin{array}{l}\text { Hyperlipidämie } \\
(\text { H_5c1) }\end{array}$ \\
\hline Intervention & 7 & 17 & 17 & 32 \\
\hline Kontrolle & 4 & 23 & 20 & 24 \\
\hline \multirow[t]{3}{*}{ Gesamt } & 11 & 40 & 37 & 56 \\
\hline & Hyperlipidämie & & & \\
\hline & $\begin{array}{c}\text { über } \\
\text { Diagnosenliste }\end{array}$ & PAVK (H_5a1) & $\begin{array}{c}\text { PAVK über } \\
\text { Diagnosenliste }\end{array}$ & $\begin{array}{c}\text { Alkoholiker } \\
\text { (H_5e1) }\end{array}$ \\
\hline Intervention & 9 & 8 & 4 & 9 \\
\hline Kontrolle & 16 & 12 & 4 & 8 \\
\hline \multirow[t]{2}{*}{ Gesamt } & 25 & 20 & 8 & 17 \\
\hline & $\begin{array}{l}\text { Alkoholiker über } \\
\text { Diagnosenliste }\end{array}$ & KHK (K_1) & $\begin{array}{c}\text { KHK über } \\
\text { Diagnosenliste }\end{array}$ & $\begin{array}{c}\text { Nikotinabusus } \\
\quad\left(\mathrm{H} \_5 \mathrm{~d} 1\right)\end{array}$ \\
\hline Intervention & 4 & 26 & 19 & 10 \\
\hline Kontrolle & 3 & 7 & 7 & 9 \\
\hline Gesamt & 7 & 33 & 26 & 19 \\
\hline
\end{tabular}

Tab.1: Abfragen der Daten aus dem Datenbanksystem.

\section{Gerinnungshemmung}

1. Qualitätsindikator: Wie viele Patienten werden mit einem Thrombozytenaggregationshemmer behandelt?

Es wurde die Anzahl der Patienten ermittelt, denen, abzüglich der Patienten mit Marcumareinnahme, ein Thrombozytenaggregationshemmer angeboten wurde.

Von 105 Patienten bekamen 86 dieses Medikament verschrieben. Von den 19 Patienten ohne Thrombozytenaggregationshemmer hatten 11 Patienten eine Kontraindikation (Dialysepatient, Alter $>65 \mathrm{~J}$, non compliance, Blutung, OP, Übelkeit), bei den übrigen acht Patienten konnte der Grund für die fehlende Gabe nicht ermittelt werden. 
2. Qualitätsindikator: Bekommen Patienten bei Vorhofflimmern und bzw. oder kardioembolischer Ätiologie des Schlaganfalls Marcumar verordnet?

Alle Patienten mit Vorhofflimmern waren mit Marcumar versorgt. Es wurden 21 Patienten mit Marcumareinnahme ermittelt, von denen 11 Patienten Vorhofflimmern bzw. eine embolische Genese des Schlaganfalls hatten.

3. Qualitätsindikator: Liegt der INR-Wert (International Normalized Ratio) bei der Gabe von Marcumar im Zielbereich (2,5-3) ?

Bei Auswertung der Zielwerteinstellung des INR bei Marcumargabe wurden die Angaben der Quick-Werte außer Acht gelassen, da sie nicht exakt in INR umrechenbar sind. Da die Patienten eine sehr unterschiedliche Anzahl von INR-Messungen erhalten haben, wurden aus statistischen Gründen nicht die Patienten, sondern die Messungen ausgewertet. Durch die große Anzahl der Messungen war ein vorher nachher Vergleich über einen Zeitraum von je 18 Monaten möglich. Vor Interventionsbeginn lagen 76 von 132 (57\%) Messungen im Zielbereich, danach 252 von 411 (61\%).

\section{Hypertonie}

4. Qualitätsindikator: Ist die Diagnose arterielle Hypertonie in der Praxisdokumentation klar erkennbar?

Die Anzahl der Patienten mit der Diagnose Hypertonie wurde aus Item H_2 (Ist bei der Patientin oder dem Patienten eine Hypertonie diagnostiziert?) ermittelt. Dies waren 96 Patienten. Davon hatten 89 Patienten einen Eintrag in der Diagnosenliste und waren somit klar dokumentiert.

5. Qualitätsindikator: Wurde bei einem Blutdruckwert größer gleich 160/100 mmHg innerhalb von drei Monaten eine Kontrollmessung durchgeführt?

Dieser Indikator gehört nach Marshall et. al. (29) zum Screening der Hypertonie. Die Daten wurden direkt über die Blutdruckwerttabelle abgefragt und eigneten sich zum vorher nachher Vergleich. Es wurde die Anzahl der Blutdruckmessungen größer gleich 160/100 mmHg gezählt, die eine Wiederholungsmessung innerhalb von drei Monaten zur Folge hatten. Die 
Tatsache, dass dadurch Patienten unterschiedlich oft mit erhöhten Werten in die Statistik eingingen, ist dabei unerheblich, da das Handeln der Hausärzte untersucht wurde. Es wurde angenommen, dass die Reaktion des Hausarztes auf einen erhöhten Blutdruck bei allen Patienten gleich ist. Die Messung des erhöhten Blutdrucks kann bis zu drei Monaten vor Interventionsbeginn liegen, wenn die Kontrollmessung nach Interventionsbeginn stattfand. Vorher wurden 541 von $684(79 \%)$ erhöhten Blutdrücken nachgemessen, nachher waren es 129 von $189(68 \%)$.

6. Qualitätsindikator: Wurden innerhalb von drei Monaten nach oder vor der Erstdiagnose der Hypertonie folgende Erkrankungen und Risikofaktoren überprüft, diagnostiziert bzw. ausgeschlossen: periphere arterielle Verschlusskrankheit (PAVK), Diabetes, Nikotinabusus, schädlicher Alkoholkonsum, Hyperlipidämie?

Wegen zu geringer Datenmenge konnte dieser Indikator für die Publikation nicht verwendet werden. Hier konnten nur 41 der Patienten mit der Diagnose Hypertonie ausgewertet werden, da für diese Abfrage ein Diagnosedatum nötig war. Drei Patienten hatten eine PAVK, vier Patienten hatten Diabetes, drei Patienten hatten eine Hyperlipidämie, bei keinem der Patienten war eine Überprüfung von Alkohol- und Nikotinabusus dokumentiert.

7. Qualitätsindikator: Wie vielen Patienten mit Zielorganschaden (Schlaganfall, PAVK, koronare Herzkrankheit (KHK) oder Diabetes), bei denen ein anhaltender (an mehr als drei hintereinander gemessenen Zeitpunkten) Blutdruck größer gleich 140/90 $m m H g$ vorliegt, ist eine medikamentöse Behandlung verordnet worden?

Diese Abfrage wurde über die Blutdruckwerttabelle und Item H_3.a. (Wann wurde die Medikation mit Antihypertensiva begonnen?) eingegeben. Nach Interventionsbeginn bekamen 10 von 12 Patienten mit drei aufeinanderfolgenden Blutdruckwerten größer gleich 140/100 mmHg ein blutdrucksenkendes Medikament verschrieben. Aufgrund der geringen Anzahl von Patienten konnte kein vorher nachher Vergleich durchgeführt werden. 
8. Qualitätsindikator: Wurden den Patienten mit diagnostizierter Hypertonie nichtmedikamentöse Maßnahmen wie Gewichtsreduktion bei BMI>30 und Begrenzung des Alkoholkonsums empfohlen?

Von 95 Patienten mit der Diagnose Hypertonie waren bei 4 Patienten Empfehlungen zur Alkoholbegrenzung dokumentiert. 8 von 95 Patienten hatten einen BMI>30. Mit 7 der 8 Patienten wurde eine Gewichtsreduktion besprochen.

9. Qualitätsindikator: Erhalten Patienten unter antihypertensiver Medikation mindestens einmal pro Jahr eine Blutdruckkontrolle?

Mit Frage H_3. (Beginn der antihypertensiven Medikation) und einer Hilfstabelle, in der die Jahreszahlen der Messungen aufgeführt sind, ließ sich dieser Qualitätsindikator untersuchen. Vor Interventionsbeginn wurden bei 46 von 77 Patienten (60\%), nach Interventionsbeginn wurden bei 65 von 77 (84\%) eine jährliche Blutdruckkontrolle durchgeführt.

10. Qualitätsindikator: Wird Patienten mit anhaltend hohen Blutdruckwerten größer 150/90 mmHg an drei oder mehr aufeinanderfolgenden Messzeitpunkten unter antihypertensiver Therapie eine Therapieänderung angeboten?

Über die Blutdrucktabelle fanden sich 19 Patienten, die über den gesamten Erhebungszeitraum unter antihypertensiver Medikation einen dreimalig erhöhten Blutdruck größer 150/90 mmHg in Folge hatten. Mittels Akteneinsicht wurden neun Patienten aus der Interventionsgruppe ermittelt, die die erhöhten Werte nach Interventionsbeginn hatten. Vier der neun Patienten wurde eine Therapieänderung empfohlen.

11. Qualitätsindikator: Wurden Nierenwerte im Zeitraum von sechs Monaten, bevor die Behandlung mit einem Angiotensin-Converting-Enzym Hemmer (ACE-Hemmer) begann, und im Zeitraum von einem Monat nach Behandlungsbeginn überprüft?

Es wurde eine Hilfstabelle angelegt, die alle verordneten Medikamente aus der Blutdrucktabelle auflistet. Anschließend wurden aus dieser Tabelle alle ACEHemmer gesammelt und den Patienten zugeordnet. Mit Hilfe der Laborliste konnten nun die Patienten gesucht werden, die im verlangten Zeitraum eine 
Untersuchung des Kreatinin- und Kaliumspiegels hatten. Von 68 Patienten mit ACE-Hemmer-Therapie bekamen acht Patienten im geforderten Zeitraum eine Kreatinin- und Kaliumkontrolle. Da sich diese acht Patienten nicht ausschließlich auf den Zeitraum nach Interventionsbeginn beziehen, wurde dieser Qualitätsindikator in der Publikation nicht verwendet.

12. Qualitätsindikator: Wurde eine Empfehlung zur Nikotinkarenz gegeben?

Durch Abfrage von Item H_5.d.1. wurden 19 Raucher ermittelt. In sechs Fällen wurde unter Item H_5.d.2. angegeben, dass eine Beratung stattfand, in der die Patienten zur Nikotinkarenz aufgefordert wurden.

\section{KHK}

13. Qualitätsindikator: Ist die Diagnose KHK in der Praxisdokumentation klar erkennbar?

Von 33 Patienten, die laut Item K_1 eine KHK haben, sind 26 in der Diagnosenliste dokumentiert.

14. Qualitätsindikator: Ist das Serumcholesterin bei KHK-Patienten in den letzten fünf Jahren kontrolliert worden?

Im Dokumentationsbogen wurde dies unter Item K_3 erfragt. Die Anzahl der KHK Patienten stammt aus Item K_1. 32 von 33 Patienten haben in den letzten fünf Jahren eine Laborkontrolle des Cholesterinwertes bekommen.

\section{KHK und Cholesterin $>200 \mathrm{mg} / \mathrm{dl}$}

15. Qualitätsindikator: Ist bei KHK Patienten, die Cholesterin Werte über $200 \mathrm{mg} / \mathrm{dl}$ hatten, eine Ernährungsberatung gemacht worden?

Die Abfrage von Item K_4.a. ergab, dass 17 von 33 KHK-Patienten Cholesterinwerte über $200 \mathrm{mg} / \mathrm{dl}$ hatten. Davon hat ein Patient in seiner Akte eine Ernährungsberatung dokumentiert. 
16. Qualitätsindikator: Bekamen diese Patienten eine medikamentöse lipidsenkende Therapie oder eine Therapieänderung, falls der Cholesterinwert trotz Medikament größer als $200 \mathrm{mg} / \mathrm{dl}$ war?

Die Abfrage von Item K_4.b.2. ermittelte vier Patienten, die ein lipidsenkendes Medikament verschrieben bekamen.

17. Qualitätsindikator: Wurde bei über sechs Monate konstant hohem Cholesterin eine Therapieänderung angeboten?

Die Abfrage von Item K_4.b.3. ermittelte drei Patienten, bei denen das Medikament umgesetzt wurde.

\section{Auswertung}

Die Zusammenstellung der Ergebnisauswertung folgt in drei Tabellen. Durch die unterschiedlich große Datenmenge der jeweiligen Qualitätsindikatoren und die wechselnde Patientenanzahl wurden mehrere statistische Tests verwendet. Bei 14 Qualitätsindikatoren wurde der Zeitraum nach Interventionsbeginn untersucht. Davon wurden sieben mit dem Fisher-Test berechnet. Bei den restlichen sechs Qualitätsindikatoren war die Datenmenge für einen statistischen Test zu klein.

Drei Qualitätsindikatoren konnten bezüglich eines Unterschiedes der Situation vor und nach Interventionsbeginn untersucht werden. Bei zwei Qualitätsindikatoren wurde ein multipler Mittelwertsvergleich durchgeführt. Ein Qualitätsindikator konnte aufgrund der gleichen Patientenzahl vor und nach Interventionsbeginn mit dem McNemar Test ausgewertet werden.

In Tabelle 2 sind die demographischen Daten und die Komorbiditäten in beiden Patientengruppen dargestellt. Ein statistisch signifikanter Unterschied wurde bei den Krankheitsbildern arterielle Hypertonie, Diabetes und KHK gefunden. Die Patienten der Interventionsgruppe litten weniger häufig an Hypertonie und Diabetes aber häufiger an KHK als die Kontrollgruppe. Bezüglich des Geschlechts, des Alters, des Body Mass Index (BMI), der Hyperlipidämie, des 
Nikotinabusus, des Alkoholkonsums und des Schädigungsgrades waren die Gruppen vergleichbar.

$$
\begin{array}{lc}
\text { Interventions- } & \text { Kontroll- } \\
\text { gruppe }(n=75) & \text { gruppe }(n=51)
\end{array}
$$

Männer/Frauen
Alter $( \pm S A)$
NIH-SS $( \pm S A)$
Hypertonie
Diabetes
Hyperlipidämie
BMI>30
PAVK
KHK
Nikotinabusus
Schädlicher Alkoholkonsum

$\begin{array}{ccc}39 / 36 & 27 / 24 & >0,999^{\mathrm{a}} \\ 69,7 \pm 13,7 & 71,5 \pm 11,0 & 0,484^{\mathrm{b}} \\ 3,5 \pm 4,7 & 6,5 \pm 14,5 & 0,101^{\mathrm{b}} \\ 52(69 \%) & 44(86 \%) & 0,034^{\mathrm{a}} \\ 17(23 \%) & 23(45 \%) & 0,011^{\mathrm{a}} \\ 32(43 \%) & 24(47 \%) & 0,716^{\mathrm{a}} \\ 7(9 \%) & 4(8 \%) & >0,999^{\mathrm{a}} \\ 8(11 \%) & 12(24 \%) & 0,080^{\mathrm{a}} \\ 26(35 \%) & 7(14 \%) & 0,013^{\mathrm{a}} \\ 10(13 \%) & 9(18 \%) & 0,614^{\mathrm{a}} \\ 9(12 \%) & 8(16 \%) & 0,601^{\mathrm{a}}\end{array}$

Tab. 2: Vergleich der demographischen Daten und schlaganfallrelevanten Risikofaktoren bzw. Komorbiditäten in Interventions- und Kontrollgruppe (Häufigkeit in der Gruppe in Klammer). SA: Standardabweichung; ${ }^{a}$ Vierfelder-Test nach Fisher; ${ }^{b}$ t-Test.

In Tabelle 3 sind die Unterschiede zwischen Interventions- und Kontrollgruppe nach Interventionsbeginn im Hinblick auf die Qualitätsindikatoren angegeben. Ein Vergleich der Situation vor und nach Interventionsbeginn ist nicht möglich, da zwei Fisher Tests nicht miteinander verglichen werden können.

Ein signifikanter Unterschied war in der Dokumentationsqualität der Diagnose Hypertonie nachweisbar. Entgegen der Erwartung war die Hypertonie bei allen Patienten der Kontrollgruppe klar erkennbar in der Patientenakte angegeben, hingegen nur bei $87 \%$ der Interventionsgruppe. Von 19 Fragestellungen bezüglich der Qualitätsindikatoren zeigten 10 keine signifikanten Unterschiede zwischen beiden Gruppen. In acht Fällen konnte aufgrund zu kleiner Zahlen kein statistischer Test durchgeführt werden. 


\section{Qualitätsindikatoren}

Antithrombotika/Antikoagulation

1.Wieviel Patienten werden

Thrombozytenaggregationshemmer verordnet? $(n=105$

Patienten)

2. Bekommen Patienten bei VHF u./o. emb. Genese

Marcumar verordnet? ( $n=11$ Patienten)

\section{Risikofaktor Hypertonie}

3. Ist die Diagnose "Hypertonie" in der

Praxisdokumentation klar erkennbar? (n=96 Patienten mit Hypertonie)

4. Wieviel Patienten bekommen bei $3 x$

$\mathrm{RR}>=140 / 100 \mathrm{mmHg}$ in Folge eine medikamentöse

Therapie angeboten? ( $n=12$ Patienten)

5.Nicht medikamentöse Maßnahmen bei Hypertonie

Empfehlung Gew.red. ( $n=8 \mathrm{BMl}>30)$

Empfehlung Alkoholbegr. ( $\mathrm{n}=95$ Patienten)

6. Bekommen Patienten mit RR>150/90mmHg $3 x$ in

Folge eine Therapieänderung? ( $n=9$ Patienten)

$\begin{array}{llc}\begin{array}{c}\text { Interventions- } \\ \text { gruppe }\end{array} & \text { Kontroll-gruppe } & \begin{array}{c}\text { Fisher-Test } \\ p\end{array} \\ 49 / 60(82 \%) & 37 / 45(82 \%) & >0,999 \\ & & -\end{array}$

$45 / 52(87 \%) \quad 44 / 44(100 \%) \quad 0,014$

$8 / 9(89 \%) \quad 2 / 3(67 \%) \quad 0,455$

$4 / 5(80 \%) \quad 3 / 3(100 \%) \quad-$

$2 / 52(4 \%) \quad 2 / 43(5 \%) \quad>0,999$

$4 / 8(50 \%) \quad 0 / 1(0 \%)$

7.Wurden innerhalb von drei Monaten nach oder vor der Erstdiagnose der Hypertonie eine PAVK, Diabetes, Nikotinabusus, schädlicher Alkoholkonsum, Hyperlipidämie überprüft? ( $n=41$ Patienten)

PAVK

Diabetes

$1 / 17(5,9 \%) \quad 2 / 24(8,3 \%) \quad>0,999$

Hyperlipidämie

$1 / 17(5,9 \%) \quad 3 / 24(12,5 \%) \quad 0,855$

Nikotinabusus

$1 / 17(5,9 \%) \quad 2 / 24(8,3 \%) \quad>0,999$

Alkohol

$0 / 17(0 \%)$

$0 / 24(0 \%)$

$0 / 17(0 \%) \quad 0 / 24(0 \%)$

8. Wurden Nierenwerte im Zeitraum von sechs

Monaten, bevor die Behandlung mit einem ACE-

Hemmer begann, und im Zeitraum von einem Monat

nach Behandlungsbeginn überprüft? ( $n=68$ Patienten)

$6 / 43(14 \%) \quad 2 / 25(8 \%) \quad 0,701$

Nikotinabusus ( $\mathrm{n}=19$ Patienten)

9. Wurde eine Empfehlung zur Karenz gegeben?

$3 / 10(30 \%) \quad 3 / 9(33 \%) \quad>0,999$

KHK ( $n=33$ Patienten)

10. Ist die Diagnose "KHK" in der Praxisdokumentation

klar erkennbar?

$19 / 26(73 \%) \quad 7 / 7(100 \%) \quad 0,299$

11. Ist das Serumcholesterin bei KHK-Patienten

innerhalb der letzten $5 \mathrm{~J}$. kontrolliert worden?

$7 / 7(100 \%) \quad>0,999$

Bei KHK und Cholesterin $>200 \mathrm{mg} / \mathrm{dl}$ (n=17Patienten)

12. Ist eine Ernährungsberatung gemacht worden?

$1 / 15(7 \%) \quad 0 / 2(0 \%)$

13. Sind lipidsenkende Medikamente verordnet worden?

$3 / 15(20 \%) \quad 1 / 2(50 \%)$

14. Wurde bei über 6 Monate konstant hohem

Cholesterin eine Therapieänderung angeboten?

$3 / 15(20 \%) \quad 0 / 2(0 \%)$

Tab. 3: Unterschiede in den Qualitätsindikatoren $(24,29)$ zwischen Interventions- und Kontrollgruppe nach Interventionsbeginn. Der p-Wert des exakten Vierfelder-Tests nach Fisher ist angegeben. Mit einem Strich sind die Vergleiche markiert, bei denen die Voraussetzungen für den Fisher-Test wegen zu kleiner Zahl der erwarteten Werte nicht erfüllt waren. 
In Tabelle 4 sind die Qualitätsindikatoren dargestellt, die einen Vergleich zwischen der Situation vor und nach Interventionsbeginn erlauben. Beim Qualitätsindikator INR-Werte im Zielbereich untersuchten wir einen Zeitraum von je 18 Monaten. Bei den beiden anderen Qualitätsindikatoren war der Zeitraum vor Intervention unbeschränkt und abhängig vom Beginn der hausärztlichen Behandlung. Das Zeitfenster nach Intervention reichte von Interventionsbeginn bis zum Zeitpunkt der Datenerhebung (18 Monate). Ein signifikanter Unterschied in die erwartete Richtung fand sich beim Indikator jährliche Blutdruckkontrolle bei Patienten mit antihypertensiver Medikation. 


\section{Qualitätsindikatoren}

\section{Antikoagulation}

INR-Werte im Zielbereich 2-3 (n=543 Messungen)
Interventionsgruppe

vorher

nachher
Vergleich

vorher

nachher

$222 / 326(68 \%)$

$7 / 16(44 \%)$

$30 / 85(35 \%)$

KGvor/nach

Gvor/nach

KGvor/IGvor

0,515

0,216

\section{Risikofaktor Hypertonie}

Kontrollmessung in 3 Mon. bei $R R \geq 160 / 100(n=873$ pathol. RR-Werte)

$68 / 109(62 \%)$

$232 / 289(80 \%)$

$61 / 80(76 \%)$

KGvor/nach

IGvor/nach

0,448

KGvor/IGvor $\quad 0,528$

Jährliche RR-Kontrolle bei antihypertensiver

Medikation ( $\mathrm{n}=77$ Patienten mit antihypertensiver

$25 / 46(54 \%)$

$42 / 46(91 \%)$

$21 / 31(68 \%)$

$23 / 31(74 \%)$

innerhalb IG

innerhalb KG

$<0,05$
n.s.

McNemar

Tab. 4: Darstellung der Qualitätsindikatoren $(24,29)$ Vergleich vor und nach Interventionsbeginn. In den Spalten rechts ist das Signifkanzniveau (wo sinnvoll) und der verwendete Test angegeben (LSD: least significant difference). 


\section{Diskussion}

Obwohl in dieser kontrollierten Interventionsstudie signifikante Unterschiede deutlich wurden, ist ein positiver Effekt von Qualitätszirkeln auf die hausärztliche Sekundärprävention nach Schlaganfall fraglich und konnte nicht eindeutig belegt werden. Im Folgenden werden die Ergebnisse im Detail analysiert.

\section{Interpretation der Daten}

\section{Zu den Abfragen der Daten/ Kritikpunkte am Dokumentationsbogen}

Einige Daten konnten problemlos abgefragt werden, andere konnten nicht über die Items des Dokumentationsbogens abgefragt werden, sondern mussten über die Untertabellen gesucht oder durch Akteneinsicht geklärt werden.

Im Folgenden sind in der Auswertung problematische Qualitätsindikatoren erwähnt, bzw. solche, die anders als geplant abgefragt werden mussten. Die Qualitätsindikatoren drei, fünf, sechs, acht, 11 und 14 sind in diesem Abschnitt nicht erwähnt, da sie ohne Schwierigkeiten abgefragt werden konnten.

Zum 1.) Qualitätsindikator: Wie viele Patienten werden mit einem Thrombozytenaggregationshemmer behandelt?

Bei den 19 Patienten, die weder Marcumar, noch einen Thrombozytenaggregationshemmer bekommen haben, muss nach Kontraindikationen gesucht werden. Die Frage nach Kontraindikationen gegen die Einnahme bzw. Applikation der genannten Medikamente ist im Dokumentationsbogen unter Item A_2.2.a.2. vermerkt. Durch den komplexen Fragenaufbau mit sechs Unterpunkten bestanden für eine maschinelle Abfrage zu viele Abhängigkeiten. Somit war diese Abfrage nicht durchführbar. Sie wurde durch Einsicht der 19 Patientenakten manuell durchgeführt. 
Zum 2.) Qualitätsindikator: Bekommen Patienten bei Vorhofflimmern und bzw. oder kardioembolischer Ätiologie des Schlaganfalls Marcumar verordnet?

Der Indikator wäre exakter und einfacher abfragbar, wenn es eine einheitliche Formulierung und Dokumentation des Krankheitsbildes gäbe. So musste man die Diagnose Vorhofflimmern an verschiedenen Orten in den Patientenakten unter verschiedenen Namen suchen. Die gleiche Diagnose Vorhofflimmern, Flimmern, VHF, TAA, absolute Tachyarrhythmie waren in der Diagnosentabelle zu finden, in wenigen Fällen aber auch nur im EKG-Befund.

Zum 4.) Qualitätsindikator: Ist die Diagnose arterielle Hypertonie in der Praxisdokumentation klar erkennbar?

Es war nicht definiert, was unter einer klaren Dokumentation zu verstehen war. Der Punkt H_2 (Ist bei der Patientin oder dem Patienten eine Hypertonie diagnostiziert?) im Dokumentationsbogen konnte bei vorhandener Diagnose in der Diagnosentabelle, aber evtl. auch bei sichtlich häufig erhöhten Blutdruckwerten des Patienten und/oder der Dokumentation einer antihypertensiven Medikamentenverordnung mit "Ja" beantwortet werden. So fanden sich 96 Patienten unter Punkt H_2, 89 Patienten hatten einen Eintrag in der Diagnosentabelle, davon 41 mit einem Datum. Es ist unklar, aus welchem Grund die wissenschaftlichen Hilfskräfte in 96 Fällen Item H_2 mit „Ja“ angekreuzt haben, obwohl die Diagnose nur bei 89 Patienten in der Diagnosenliste zu finden war. Die Anzahl der Patienten mit Hypertonie ist somit abhängig von den Einträgen der wissenschaftlichen Hilfskräfte.

Zum 7. Qualitätsindikator: Wie vielen Patienten mit Zielorganschaden (Schlaganfall, PAVK, koronare Herzkrankheit (KHK) oder Diabetes), bei denen ein anhaltender (an mehr als drei hintereinander gemessenen Zeitpunkten) Blutdruck größer gleich 140/90 mmHg vorliegt, ist eine medikamentöse Behandlung angeboten worden?

Er ist unter Item H_8. und H_8.c im Dokumentationsbogen zu finden. Da aber nicht vermerkt ist, dass die erhöhten Messungen hintereinander stattfinden mussten, lieferte die Abfrage falsch große Zahlen. So wurde diese Abfrage über 
die Blutdruckwerttabelle und Item H_3.a. (Wann wurde die Medikation mit Antihypertensiva begonnen?) eingegeben.

Zum 9. Qualitätsindikator: Erhalten Patienten unter antihypertensiver Medikation mindestens einmal pro Jahr eine Blutdruckkontrolle?

Problematisch ist hier die kurze Zeitspanne nach Interventionsbeginn. Während der Zeitraum vor Interventionsbeginn vom Beginn der hausärztlichen Betreuung abhing und dadurch bis zu 20 Daten lieferte, war der Zeitraum zwischen Interventionsbeginn und Datenerhebung mit zwei Jahren relativ kurz.

Zum 10. Qualitätsindikator: Wird Patienten mit anhaltend hohen Blutdruckwerten größer 150/90 mmHg an drei oder mehr aufeinanderfolgenden Messzeitpunkten unter antihypertensiver Therapie eine Therapieänderung angeboten?

Da wie bei Qualitätsindikator sieben im Dokumentationsbogen nicht gefordert wurde, dass die Werte hintereinander gemessen sein sollen, konnte die Abfrage nicht über H_12. (Therapieänderung) durchgeführt werden.

Zum 12. Qualitätsindikator: Wurde eine Empfehlung zur Nikotinkarenz gegeben? Der wichtige Risikofaktor Nikotinabusus wird selten von den Ärzten dokumentiert. Daher war die Anzahl der Beratungen mit sechs Patienten klein.

Zum 13. Qualitätsindikator: Ist die Diagnose KHK in der Praxisdokumentation klar erkennbar?

Es war hier wie bei der Diagnose Hypertonie nicht eindeutig definiert, was darunter zu verstehen war. Unter Item K_1 wurde die Diagnose KHK festgehalten. Die Anzahl der Patienten, die laut Item K_1 an einer KHK litten, waren nicht deckungsgleich mit der Diagnosenliste. Daher wurden die Patienten, die in der Diagnosenliste einen Eintrag hatten als „klare Dokumentation" definiert. Sieben Patienten wurde in Item K_1 eine KHK bescheinigt, die aber in der Diagnosenliste gar nicht dokumentiert war. Eine Erklärung dafür könnte sein, dass die wissenschaftlichen Hilfskräfte die Diagnose aus Arztbefunden (z. B. Herzkatheterbefund) übernommen haben. 
Zum 15. Qualitätsindikator: Ist bei KHK Patienten, die Cholesterin Werte über 200 $\mathrm{mg} /$ dl hatten, eine Ernährungsberatung durchgeführtt worden?

Ein Patient von 17 hat eine Ernährungsberatung bekommen. Hier muss daran gedacht werden, dass Beratungen von Hausärzten in der Regel nicht immer schriftlich dokumentiert werden. Es ist wahrscheinlich, dass viele oder alle die Beratung bekommen haben, sie aber schriftlich nicht fixiert wurde.

\section{Zu der Ergebnissauswertung}

Die beiden Patientengruppen unterschieden sich im Hinblick auf die Komorbidität (vgl. Tab. 1). Auffallend ist weiterhin ein recht unterschiedlicher Schädigungsgrad (NIH-SS) in beiden Gruppen. Er ist jedoch statistisch nicht signifikant und kommt durch die große Streuung zustande. Wenige stark geschädigte Patienten in der Kontrollgruppe haben den durchschnittlichen Schädigungsgrad ansteigen lassen. Insgesamt gesehen war jedoch in beiden Patientengruppen die Gesamtzahl der relevanten Risikofaktoren vergleichbar. Ein störender Einfluss auf das Studienergebnis ist daher unwahrscheinlich.

Bei den Qualitätsindikatoren der Tabelle 3 fanden sich bis auf die Dokumentationsqualität der Diagnose Hypertonie keine Unterschiede zwischen den Gruppen. Entgegen der Erwartung war die Dokumentationsqualität in der Kontrollgruppe signifikant besser als in der Interventionsgruppe.

Bei den Qualitätsindikatoren der Tabelle 4 fanden sich bei allen drei aufgeführten Indikatoren signifikante Unterschiede zwischen Interventions- und Kontrollgruppe. Es muss berücksichtigt werden, dass bei zwei von drei Indikatoren die Fallzahl nicht durch die Zahl der Patienten, sondern durch die Zahl der aus hausärztlicher Sicht interventionsbedürftigen Ereignisse bzw. Therapieerfolge bestimmt war. Bei dem Item INR im Zielbereich gab es einen signifikanten Unterschied Interventions- vs. Kontrollgruppe nach Interventionsbeginn. Allerdings unterschieden sich beide Gruppen schon in der Ausgangssituation (nicht signifikant). In der Kontrollgruppe verschlechterte sich die Quote der INR-Werte im Zielbereich, wohingegen sie sich in der Inter- 
ventionsgruppe verbesserte. Da die Verschlechterung in der Kontrollgruppe nicht plausibel erklärt werden kann ist fraglich, ob der signifikante Unterschied zwischen Intervention und Kontrolle nach Interventionsbeginn ausschließlich auf die Intervention zurückzuführen ist.

Beim zweiten Item, der Kontrollmessung bei pathologischen Blutdruckwerten, findet sich ein signifikanter Unterschied, jedoch entgegen der Erwartung.

Bei den Blutdruck-Kontrollen bei antihypertensiver Medikation ist ein Interventionseffekt nachweisbar. In der Interventionsgruppe waren signifikante Verbesserungen $\mathrm{zu}$ beobachten, die in der Kontrollgruppe nicht auftraten. Diese Beobachtung ist deutlich und plausibel, da sie Gegenstand der Qualitätszirkelarbeit war.

In der Gesamtsicht muss festgestellt werden, dass sich signifikante Unterschiede zwischen Intervention und Kontrolle nur da nachweisen ließen, wo ein Vergleich vor vs. nach Interventionsbeginn möglich war. Aus methodischen Gründen war das nur bei einem Teil der Items bzw. Indikatoren möglich (s.u.). Eine weitere Schwierigkeit bestand darin, dass mögliche positive Effekte in der Kontrollgruppe allein durch die Teilnahme an der Studie nicht ausgeschlossen werden können. Die Möglichkeit, dass die Hausärzte der Kontrollgruppe ihr Verhalten aufgrund des Bewusstseins, an dieser Studie teilzunehmen, änderten, nennt man Hawthorneeffekt.

Angesichts dieser methodischen Schwierigkeiten stellt sich die Frage, ob Qualitätszirkel tatsächlich keinen günstigen Effekt auf die hausärztliche Sekundärprävention nach Schlaganfall haben oder ob ein positiver Aspekt aufgrund des Studiendesigns oder der Studiendurchführung nicht nachgewiesen werden konnte. Der zweite Aspekt wird nachfolgend detailliert diskutiert.

\section{Kritikpunkte und Limitationen}

Datenqualität: Wir mussten feststellen, dass das Qualitätsindikatorenset nicht an die hausärztliche Realität angepasst war. Viele der benötigten Informationen konnten in den Praxisdokumentationen nicht ermittelt werden. Dies hat verschiedene Gründe. Die Qualitätsindikatoren Empfehlung Nikotin- und 
Alkoholkarenz sowie Beratungsgespräche über Ernährung und Gewichtsreduktion werden in der Regel vom Hausarzt nicht dokumentiert. Eine weitere Schwierigkeit stellte die uneinheitliche Dokumentation dar. In Papierakten fehlte häufig ein Datum. Daraus folgte, dass zu einem handschriftlich notierten Blutdruck die elektronisch gespeicherte Medikamentenverordnung nicht zuzuordnen war. Aufgrund der geringen Menge der Daten mit Datumsangabe ließen sich nur bei drei Qualitätsindikatoren vorher/nachher Vergleiche durchführen.

Studiendesign: Die ursprünglich geplante Studie mit der Powerkalkulation von 400 Patienten konnte aus organisatorischen Gründen nicht durchgeführt werden. Für die abgewandelte Studienform wurde keine erneute Powerkalkulation durchgeführt. Daher spiegelt die Studie eine Prozessqualität wider und hat bei vielen Indikatoren zu geringe Fallzahlen. Weiterhin wäre eine Randomisierung wünschenswert gewesen, aber dies war aufgrund der großen Entfernungen der jeweiligen Praxis vom Ort des Qualitätszirkels im ländlichen Studiengebiet nicht durchführbar. Daher erfolgte die Gruppeneinteilung a priori anhand des Praxisortes. Zuletzt muss bedacht werden, dass ein möglicher günstiger Effekt der Qualitätszirkelarbeit durch den vergleichsweise kürzeren Zeitraum nach Interventionsbeginn verwischt werden konnte.

Studiendurchführung: Die Dokumentation der Patientendaten erfolgte auf Papier in den Praxen und wurde anschließend in die elektronische Datenbank eingegeben. Durch diesen Zwischenschritt können Übertragungsfehler nicht ausgeschlossen werden.

Zusammenfassend muss festgestellt werden, dass mit dieser Studie viel mehr die Dokumentationsqualität in den Hausarztpraxen statt der Behandlungsqualität untersucht wurde.

\section{Qualitätszirkel und Versorgungsforschung}

Vor dem Hintergrund limitierter Ressourcen im Gesundheitswesen gewinnen präventive Aspekte gegenüber der Therapie immer mehr Bedeutung. Allgemein wird versucht, die Struktur- und Prozessqualität der Prävention (und der Therapie) durch die Entwicklung von Leitlinien zu verbessern. 
Leitlinien sollen Hausärzten helfen, die Betreuung des einzelnen Patienten an allgemein anerkannten wissenschaftlichen Erkenntnissen auszurichten. Allerdings werden Leitlinien nicht ausreichend implementiert (15, 21). Hindernd wirken die Existenz verschiedener Leitlinien $\mathrm{zu}$ identischen Versorgungsproblemen, eine häufig nicht beurteilbare Interessenlage der Autorenschaft, fehlende Kosten- und Nutzenkalkulation und die unzureichende Evidenz vieler Leitlinien. Dies führt $\mathrm{zu}$ mangelnder Praktikabilität und geringer hausärztlicher Relevanz der Leitlinien. Außerdem wird den Hausärzten zu wenig Raum für eigene ärztliche Entscheidungen gelassen (12, 17, 26). Qualitätszirkel sind ein wertvolles Instrument, um die Lücke zwischen den eher theoretisch orientierten Leitlinien und der Praxis zu überwinden.

Im Bereich der Allgemeinmedizin konnten signifikante Effekte der Qualitätszirkelarbeit nachgewiesen werden (4). Im Vergleich zur Situation vor Beginn der Intervention profitierten die Hausärzte von ihrer Qualitätszirkelarbeit durch bessere kollegiale Beziehungen, einen Nutzen für die Praxisroutine, eine insgesamt höhere Berufszufriedenheit, eine erhöhte Mitarbeitermotivation und einen Austausch über berufspolitische Themen.

Im Herbst letzten Jahres wurde ein Nachweis erbracht, der zeigt, dass es mit einem gezielten Aktionsprogramm, welches unter anderem auf Qualitätszirkelarbeit gestützt war, gelungen ist, die nationalen Leitlinien zum Thema Depression im deutschen Gesundheitssystem umzusetzen (19). Nachdem im deutschen Clearingverfahren evidenz-basierte nationale Leitlinien entwickelt worden sind (11), wurde mit einem speziellen Implementierungsprogramm begonnen, diese in die Praxis umzusetzen. Die wichtigsten Schritte dabei sind die Entwicklung eines Dokumentationssystems, eine kontinuierliche Fortbildung der Ärzte in interdisziplinären Qualitätszirkeln und die Information der Öffentlichkeit über die Erkrankung $(18,19)$.

In Qualitätszirkeln können sich die Hausärzte mit den Leitlinien produktiv auseinandersetzen, um diese in ihre tägliche Praxis zu „übersetzen.“ In der vorliegenden Arbeit wurde versucht, diesen möglichen Vorteil der 
Qualitätszirkel in der hausärztlichen Praxis zu belegen. Leider ist dieser Nachweis nicht gelungen. Möglicherweise liegt das daran, dass die Prozessqualität der Sekundärprävention nach Schlaganfall ohnehin schon relativ hoch ist.

Es stellt sich jedoch insgesamt die Frage, welchen Stellenwert Qualitätszirkel als sinnvolles Instrument einnehmen, um Leitlinien in die hausärztliche Praxis zu implementieren. Vielleicht waren die oben erwähnten methodischen Schwierigkeiten Grund dafür, dass in unserer Studie ein positiver Effekt von Qualitätszirkeln nicht nachgewiesen werden konnte.

In diesem Kontext ist von Bedeutung, dass die Versorgungsforschung ganz allgemein trotz ihrer hohen Relevanz offenbar noch mit erheblichen methodischen Schwierigkeiten kämpft (16). Wie oben diskutiert, kann auch bei der vorliegenden Arbeit nicht ausgeschlossen werden, dass das Ergebnis zum Teil durch methodische Mängel verursacht wurde. Für diese Studie wurde ein großer Aufwand betrieben, der ein vergleichsweise kleines Ergebnis zeigt. Ein besser praktikables Indikatorenset und die breite Einführung einer einheitlichen elektronischen Dokumentation wären erste Ansatzpunkte zur Verbesserung der diesbezüglichen Versorgungsforschung. Damit könnte dann vielleicht die Frage beantwortet werden, inwieweit Qualitätszirkel zur Implementierung von Leitlinien in die hausärztliche Praxis beitragen und damit die Versorgungsqualität verbessern können.

\section{Schlussfolgerung}

In der vorgelegten kontrollierten Interventionsstudie konnte ein Vorteil von Qualitätszirkeln zur Verbesserung der Sekundärprävention nach Schlaganfall nicht belegt werden. Eine entscheidende Schwierigkeit dabei war die uneinheitliche, oft papiergestützte Dokumentation in den hausärztlichen Praxen. Daher wäre die Entwicklung und breite Einführung einer einheitlichen und unkompliziert handhabbaren Software zur Praxisdokumentation wünschenswert.

Eine Ineffizienz von Qualitätszirkeln bei der Sekundärprävention nach Schlaganfall und auch ganz generell, kann aus unserer Studie nicht abgeleitet werden. 
Weitere, methodisch ausgefeiltere Untersuchungen müssen klären, ob Qualitätszirkel ein taugliches Instrument zur Verbesserung der Sekundärprävention nach Schlaganfall sind. 


\section{Zusammenfassung}

Hintergrund/Ziele: In der vorliegenden kontrollierten Interventionsstudie wurde der Effekt der Qualitätszirkelarbeit auf die hausärztliche Sekundärprävention nach Schlaganfall untersucht.

Methoden: Es wurden 212 Patienten aus vier Akutkrankenhäusern im Südschwarzwald nach einem ersten Schlaganfall im Zeitraum 15.11.2000-31.3.2002 für die Studie rekrutiert. Ein Teil der betreuenden Hausärzte nahm zwischen März 2002 und März 2003 an Qualitätszirkeln teil, die sich mit der Sekundärprävention nach Schlaganfall beschäftigten. Die Zuteilung der Hausärzte zur Interventions- bzw. Kontrollgruppe erfolgte nach dem Ort der Praxis. Die Versorgungsqualität der Patienten wurde an Hand vorher entwickelter Qualitätsindikatoren beurteilt. Insgesamt wurden die Daten von 126 Patienten ausgewertet. Der statistische Vergleich erfolgte mit dem exakten Vierfeldertest nach Fisher, dem t-Test für ungepaarte Vergleiche, dem McNemar-Test und multiplen Mittelwertvergleichen ( $<<0,05)$.

Ergebnisse: 75 Patienten wurden der Interventionsgruppe zugeteilt und von 36 Hausärzten betreut. Die 51 Patienten der Kontrollgruppe wurden von 25 Hausärzten betreut. Die beiden Gruppen waren in der Summe aller Merkmale vergleichbar, zeigten aber Unterschiede in der Häufigkeit bestimmter Komorbiditäten. Statistisch signifikante Unterschiede konnten nur für die Einstellung des INR bei Antikoagulation und die Behandlungsqualität des wichtigen Risikofaktors Hypertonie nachgewiesen werden. Der Anteil der antihypertensiv behandelten Patienten, bei denen jährlich Blutdruckkontrollen vorgenommen wurden, stieg in der Interventionsgruppe nach Interventionsbeginn an. In der Kontrollgruppe wurde dies nicht beobachtet. Jedoch wurden in der Interventionsgruppe nach Interventionsbeginn Blutdruckerhöhungen bei Patienten mit arterieller Hypertonie seltener adäquat nachuntersucht als vorher. In der Kontrollgruppe war keine Veränderung zu verzeichnen.

Schlussfolgerungen: Eindeutige systematische Effekte der Qualitätszirkelarbeit auf die hausärztliche Sekundärprävention nach Schlaganfall konnten an dem untersuchten Hausarztkollektiv nicht belegt werden. 


\section{Literatur}

1. Adams HPJ, Bendixen BH, Kappelle LJ, Biller J, Love BB, Gordon DL and Marsh EE (1993) Classification of subtype of acute ischemic stroke. Definitions for use in a multicenter clinical trial. TOAST. Trial of Org 10172 in Acute Stroke Treatment. Stroke 24: 35-41

2. Ärztliche Zentralstelle Qualitätssicherung (2001) Beurteilung klinischer Messgrößen des Qualitätsmanagements - Qualitätskriterien und Indikatoren in der Gesundheitsversorgung - Konsenspapier der Bundesärztekammer, der Kassenärztlichen Bundesvereinigung und der AWMF. äzq Ärztliche Zentralstelle Qualitätssicherung,

3. Berger K, Weltermann B, Kolominsky-Rabas P, Meves S, Heuschmann P, Bhoner J, Neundorfer B, Hense HW and Buttner T (1999) The reliability of stroke scales. The german version of NIHSS, ESS and Rankin scales. Fortschr Neurol Psychiatr 67: 81-93

4. Berndt D, Tausch B and Härter M (2001) Perceived effectiveness of diagnostic and therapeutic guidelines in primary care quality circles. International Journal for Quality in Health Care 13: 239-246

5. Bloch RE, Lauterbach K, Oesingmann U, Rienhoff O, Schirmer HD and Schwartz FW (1997) Beurteilungskriterien für Leitlinien in der medizinischen Versorgung. Beschlüsse der Vorstände von Bundesärztekammer und Kassenärztlicher Bundesvereinigung, Juni 1997. Deutsches Ärzteblatt 33: 2154-2155

6. Brott T, Adams HPJ, Olinger CP, Marler JR, Barsan WG, Biller J, Spilker J, Holleran R, Eberle R, Hertzberg V and Walker M (1989) Measurements of acute cerebral infarction: a clinical examination scale. Stroke 20: 864870

7. Centers for Disease Control (2003) Hospitalizations for stroke among adults aged over 65 years - United States, 2000. JAMA 290: 1023-1024

8. Diener HC (2005) 10 Jahre Stroke Units in Deutschland. Akt Neurol 32: 311-312

9. $\quad$ Dobkin BH (2005) Rehabilitation after stroke. N Engl J Med 352: 16771684

10. Expertenkreis Schlaganfall des Deutschen Leitlinien-Clearingverfahrens (2005) Leitlinien-Clearingverfahren Schlaganfall: Übersicht und Zusammenfassung. Zusammenfassung und Empfehlungen für eine nationale Leitlinie "Schlaganfall" für Deutschland. http:/ / www.leitlinien.de/clearingverfahren/index/clearingberichte/in dex/schlaganfall/00schlaganfall/08schlaganfall/view Zugriff: 1.2.2006

11. Expertenkreises Depression des Deutschen Leitlinien-Clearingverfahrens (2003) Leitlinien-Clearingverfahren Depression. 
http:// www.leitlinien.de/ clearingverfahren/clearingberichte/depressio n/00depression/7depression/view\# volltext Zugriff 12.12.2005

12. Ferber L and Ferber C (2004) Beziehungen zwischen "Autoren" und "Adressaten" von Leitlinien-ein vernachlässigtes Thema bei der Implementation von Leitlinien? Gesundheitswesen 66:

13. Field MJ and Lohr KN (1990) Clinical practice guidelines - directions for a new program. National Academy Press, Washington D.C.

14. Forster DH, Krause G, Gastmeier P, Ebner W, Rath A, Wischnewski N, Lacour M, Ruden H and Daschner FD (2000) Can quality circles improve hospital-aquired infection control? Journal of Hospital Infection 45: 302310

15. Goldberg HI, Wagner EH, Fihn SD, Martin DP, Horowitz Cr, Christensen DB, Cheadle AD, Diehr P and Simon G (1998) A randomized trial of CQI teams and academic detailing: can they alter compliance with guidelines? Joint Commission Journal on Quality Improvement 24: 130-142

16. Grimshaw JM, Thomas RE, MacLennan G, Fraser C, Ramsay CR, Vale L, Whitty P, Eccles MP, Matowe L, Shirran L, Wensing M, Dijkstra R and Donaldson C (2004) Effectiveness and efficiency of guideline disseminationa and implementation strategies. Health Technol Assess 8: iii-iv, 1-72

17. Grol R, Dalhuijsen J, Siep T, T`Veld C, Rutten G and Mokkink H (1998) Attributes of clinical guidelines that influence use of guidelines in general practice: observational study. BMJ 317: 858-861

18. Härter M, Bermejo I, Kratz S and Schneider F (2003) Continuing education and quality management measures for the implementation of clinical practice guidelines. Z Ärztl Fortbildung Qualitätssicherung 97: 67-73

19. Härter M, Bermejo I, Ollenschläger G, Schneider F, Gaebel W, Hegerl U, Niebling W and Berger M (2005) Improving quality of care for depression: the German Action Programme for the implementation of evidence-based guidelines. International Journal for Quality in Health Care 1-7

20. Härter M and Tausch B (2004) Anspruch und Wirklichkeit von Qualitätszirkeln in der ambulanten Versorgung. Bundesgesundheitsblatt Gesundheitsforschung Gesundheitsschutz 118-124

21. Hasenbein U, Frank B and Wallesch CW (2003) Die Akzeptanz von Leitlinien und Problemen bei ihrer Implementierung. Akt Neurol 30: 451-461

22. Hendricks HT, van Limbeek J, Geurts AC and Zwarts MJ (2002) Motor recovery after stroke: a systematic review. Arch Phys Med Rehabil 83: 1629-1637 
23. Hetzel A (2000) What is the Standard in Diagnostics, Therapy and Prophylaxis of Cerebral Ischemia? Hämostasiologie 20: 70-76

24. Jahn H, Hetzel A, Luckner A and Niebling W (2003) Entwicklung von Qualitätsindikatoren (QIs) zur Evaluation hausärztlicher Sekundärprävention nach Schlaganfall. Z Allg Med 79: 38

25. Jorgensen $\mathrm{H}$, Stig M, PhD, Nakayama $\mathrm{H}, \mathrm{MD}, \mathrm{PhD}$, Raaschou $\mathrm{H}$ and Olsen T (1997) Akute stroke: Prognosis and aprediction of the effect of medical treatment on outcome and health care utilization: The Copenhagen Stroke Study. American Academy of Neurology 49: 13351342

26. Kirchner H, Fiene $M$ and Ollenschläger G (2003) Bewertung und Implementierung von Leitlinien. Rehabilitation 42: 74-82

27. MacMahon S, Peto R, Cutler J, Collins R, Sorlie P, Neaton J, Abbott R, Godwin J, Dyer A and Stamler J (1990) Blood pressure, stroke and coronary heart disease.Part 1, prolonged differences in blood pressure: prospective observational studies corrected for the regression dilution bias. Lancet 335: 765-774

28. Mahoney F and Barthel D (1965) Functional evaluation: the Barthel Index. Maryland State Med Journal 14: 61-65

29. Marshall M (2002) Quality indicators for general practice. Royal Society of Medicine Press, Manchester,

30. McGlynn EA, Asch SM, Adams J, Keesey J, Hicks J, DeCristofaro A and Kerr EA (2003) The Quality of Health Care Delivered to Adults in the United States. N Engl J Med 348: 2635-2645

31. Rankin J (1957) Cerebral vascular accidents in patients over the age of 60 . Scott Med J 2: 254-268

32. Sachs L (1997) Angewandte Statistik: Anwendung statistischer Methoden. 8.Aufl. Springer Berlin Heidelberg,

33. Weisser P, Härter M and Tausch B (2000) Hausärztliche Qualitätszirkel zwischen Anspruch und Wirklichkeit - eine Interaktionsanalyse. Z ärztl Fortbild Qualsich 94: 4-10

34. Wensing M, Broge B, Kaufmann-Kolle P, Andres E and Szecsenyi J (2004) Quality circles to improve prescribing patterns in primary medical care: what is their actual impact? Journal of Evaluation in Clinical Practice 10: 457-466

35. Wolf PA (2001) Hypertension. In: Hachinski V (eds.) Stroke prevention. Aufl. Oxford University Press, Oxford, New York 


\section{Abkürzungen}

ACE-Hemmer:

ÄZQ:

BÄK:

BMI:

EBM:

EKG:

INR:

KBV:

KHK:

LSD:

MRS:

NIH-SS:

PAVK:

RR

QZ:

TAA:

TIA:
Angiotensin-Converting-Enzym Hemmer

Ärztliche Zentralstelle für Qualitätssicherung

Bundesärztekammer

Body Mass Index

Evidence based medicine

Elektrokardiogramm

International Normalized Ratio

Kassenärztliche Bundesvereinigung

Koronare Herzkrankheit

Least Significant Difference

Modifizierte Rankin Skala

National Institutes of Health Stroke Scale

Periphere arterielle Verschlusskrankheit

Indirekte Blutdruckmessung nach Riva Rocci

Qualitätszirkel

Tachyarrhythmia absoluta

Transitorische Ischämische Attacke

TOAST-Klassifikation: Trial of Org 10172 in Acute Stroke Treatment VHF: Vorhofflimmern 


\section{Danksagung}

Die Seite 42 (Danksagung) enthalten

persönliche Daten. Sie sind deshalb nicht Bestandteil der Online-Veröffentlichung 


\section{Lebenslauf}

Die Seiten 43-44 (Lebenslauf) enthalten persönliche Daten. Sie sind deshalb nicht Bestandteil der Online-Veröffentlichung 
- $44-$ 
Anhang

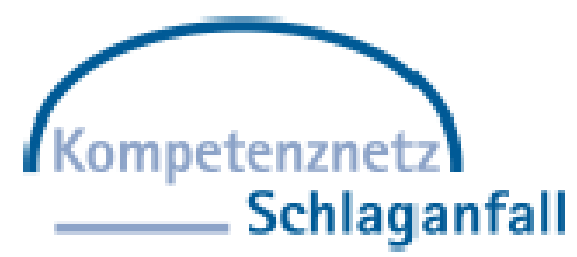

Dokumentationsbogen zur Erhebung von

Qualitätsindikatoren zur hausärztlichen

Sekundärprävention nach Schlaganfall

Patienten ID

Hausarzt ID:

Datum der vorliegenden Dokumentation: 


\section{Ausfüllanleitung}

Die Daten, die zum ausfüllen dieses Dokumentationsbogens nötig sind, werden aus den Patientenakten der Hausärzte entnommen. Diese Patientenakten umfassen alle den Patienten betreffende Dokumentationen. Also: Handschriftliches, Briefe aus Kliniken, fachärztliche Befunde und die in der Praxis-EDV gespeicherten Daten.

Der Fragebogen ist in 4 Abschnitte gegliedert:

$E_{-\ldots}$ Einführung

A_... Items zu Antithrombose und Antikoagulation

H_... Items zu Hypertonie

K_... Items zu KHK und Cholesterin

Wichtig ist, dass von jedem Item eine Antwort angekreuzt oder angegeben wird.

Die Fragen sind hierarchisch in Fragekomplexe gegliedert. Die Gliederung besteht aus folgendem Aufbau: X_1.a.1.1.1. Um die Fragen verständlich zu halten, wurde versucht diese "Fragebäume" möglichst kurz zu halten. Die Inhalte der in der Hierarchie untergeordneten Fragen beziehen sich jeweils auf die direkt übergeordneten Inhalte.

Beispiel:

1.a. Ist die Therapie zur antithrombotischen Behandlung wie bei Entlassung aus dem Krankenhaus empfohlen? (vergl. Arztbrief des Klinikaufenthaltes wegen Schlaganfall) Ja o Nein o keine Angabe möglich $\theta$

1.a.1. Wenn Nein, - dieses Nein bezieht sich auf die Antwortmöglichkeit in

Frage 1.a. - wurde die Therapie durch den Hausarzt oder einen anderen behandelnden Arzt abgesetzt? Ja o Nein o keine Angabe möglich $\theta$

1.a.1.1. Wenn Ja, wann?

- dieses Ja bezieht sich auf die Antwortmöglichkeit in Frage

1.a.1.

1.a.1.2. Wenn Ja, lag eine der unten angefügten Kontraindikationen $(\mathrm{KI})^{1}$ vor? Ja o Nein o keine Angabe möglich $\theta$,

Dieses Ja bezieht sich ebenfalls auf die Antwortmöglichkeit in Frage 1.a.1., die Frage 1.a.1.2. ist der Frage 1.a.1.1. in der Hierarchie gleichgestellt.

\footnotetext{
${ }^{1} \mathrm{KI}$, absolute: nachgewiesene zerebrale oder extrazerebrale Blutung, schlecht eingestellter Hypertonus, ausgeprägte zerebrale Mikroangiopathie im CT, hämorrhagische Diathese, schwere Leber-/Nierenschäden (Rest$\mathrm{N}>60 \mathrm{mg} \%$ ), Endokarditis lenta, Gravidität, frisch operierter Pat.

${ }^{*} \mathrm{KI}$, relative: schwere arterielle Hypertonie, zerebrale Mikroangiopathie, schlecht kontrollierter IDDM, dekompensierte Herzinsuffizienz, Non-Compliance, Alter $>65$ Jahre, laufende Behandlung mit gerinnungshemmenden Substanzen.
} 


\section{Einführung}

E_1. Ist der Patient verstorben Ja o Nein o $\quad$ keine Angabe möglich $\theta$

E_1.a.Wenn ja wann:

Datum

E_1.b. Wenn ja, was war die Todesursache:

E_2. Welche Diagnosen liegen vor?

Bitte alle Diagnosen aufführen und das Datum der Erstdiagnose, bzw. falls dieses nicht vorliegt, das Datum der erstmaligen Dokumentation der Diagnose dazu notieren. Falls gesondert gekennzeichnet, bitte auch notieren, ob es sich um eine Dauerdiagnose handelt.

Bitte alles in der Diagnosentabelle aufführen

\section{Tabelle 1: Diagnosentabelle}

\begin{tabular}{lll}
\hline Diagnose & Datum & Als Dauerdiagnose \\
& gekennzeichnet? \\
\hline
\end{tabular}

Die Tabelle muss bei Bedarf fortgesetzt werden. 


\section{Antikoagulation \& Antithrombose}

Qualitätsindikatoren (QI)-Dokumentationsbogen zur antikoagulatorischen und antithrombotischen Therapie

A_1. Welches antithrombotische/antikoagulatorische Medikament erhält der Patient aktuell?:

1. ASS (z.B. Aspirin, Godamed)

2. Ticlopidin (z.B. Tyklid)

3. Clopidogrel (z.B. Iscover, Plavix)

4. Dipyridamol (z.B. Persantin)

5. ASS/Dipyridamol (z.B. Aggrenox)

7. Phenprocoumon

(Marcumar)

8. Heparin

A_2. Ist die Therapie zur antithrombotischen Behandlung wie bei Entlassung aus dem Krankenhaus empfohlen? (vergl. Arztbrief des Klinikaufenthaltes wegen Schlaganfall)

Ja o Nein o Keine Angabe möglich $\theta$

2.a. Wenn Nein, wurde die Therapie durch den Hausarzt oder einen anderen behandelnden Arzt abgesetzt? Ja o Nein o keine Angabe möglich $\theta$

2.a.1. Wenn Ja, wann?

Datum

2.a.2. Wenn Ja, lag eine der unten angefügten Kontraindikationen $(\mathrm{KI})^{2}$ vor? Ja o Nein o keine Angabe möglich $\theta$

2.a.2.1. Wenn Ja, welche? KI

2.a.2.2. Wenn Nein, sind sonstige Gründe für ein absetzen der Therapie angegeben?

Ja o Nein o

2.a.2.2.1. Wenn Ja, welche?

Gründe

2.b.Wurde ein anderes Medikament zur Antikoagulation \& Antithrombose angesetzt? Ja o Nein o

2.b.1. Wenn Ja, wann?

Datum

${ }^{2} \mathrm{KI}$, absolute: nachgewiesene zerebrale oder extrazerebrale Blutung, schlecht eingestellter Hypertonus, ausgeprägte zerebrale Mikroangiopathie im CT, hämorrhagische Diathese, schwere Leber-/Nierenschäden (Rest$\mathrm{N}>60 \mathrm{mg} \%$ ), Endokarditis lenta, Gravidität, frisch operierter Pat.

* KI, relative: schwere arterielle Hypertonie, zerebrale Mikroangiopathie, schlecht kontrollierter IDDM, dekompensierte Herzinsuffizienz, Non-Compliance, Alter $>65$ Jahre, laufende Behandlung mit gerinnungshemmenden Substanzen. 
2.b.2. Wenn Ja, welches Medikament?

2.b.3. In welcher Dosierung?

A_3. Wenn die/der Patientin/Patient Marcumar einnimmt, ist der Ziel-INR (der empfohlene INR-Wertebereich) angegeben?

Ja o Nein o

3.a. Wenn Ja, was ist der Ziel-INR?

3.b. Wo ist der Ziel-INR angegeben?

3.c. Im Arztbrief der primärversorgenden Klinik? Ja o Nein o 3.c.1. Wenn Ja, wann? Datum

3.d. Im Arztbrief der Rehabilitationsklinik? Ja o Nein o

3.d.1. Wenn Ja, wann? Datum

3.e. Im Arztbrief des niedergelassenen Kardiologen oder Internisten? Ja o Nein o

3.e.1. Wenn Ja, wann? Datum

3.f. Im Arztbrief des niedergelassenen Neurologen? Ja o Nein o

3.f.1. Wenn Ja, wann? Datum 
A_4. Wie hoch ist der INR im Verlauf der hausärztlichen Betreuung?

Zur Beantwortung der Frage, bitte alle(!) seit Entlassung aus dem Krankenhaus gemessenen Werte in der Datum-INR-Tabelle aufführen. Bitte immer den INR dokumentieren, nur falls der INR nicht dokumentiert ist, den Quick dokumentieren

Tabelle 2: Datum-INR-Tabelle

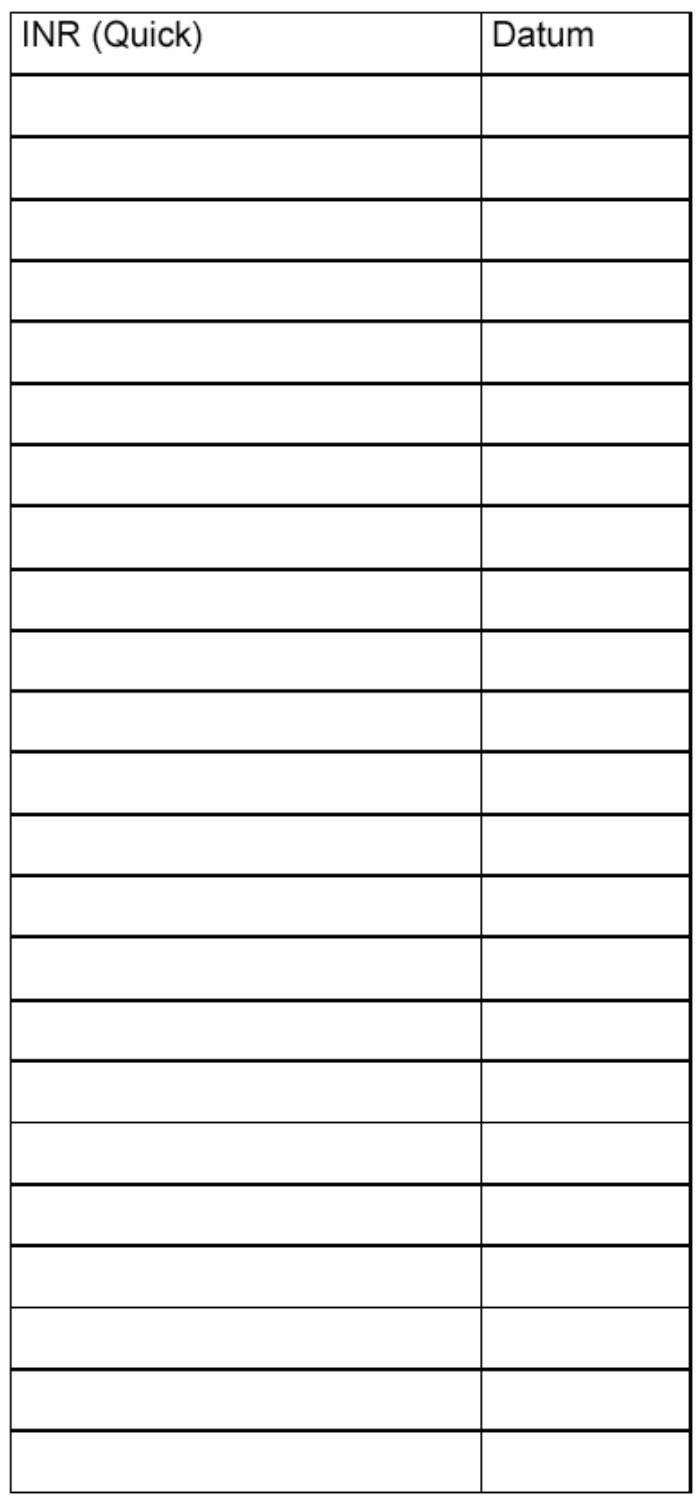

Die Tabelle muss bei Bedarf fortgesetzt werden. 
A_5. Falls der INR nicht in empfohlenem Bereich liegt. Sind Kontraindikationen (KI) gegen Antikoagulation dokumentiert?

5.a. Wenn Ja, welche KI?

\section{H_5.d. Nikotinabusus}

5.d.1. Liegt bei der/dem Patientin/Patienten ein Nikotinabusus vor? Ja o Nein o unbekannt $\theta$

5.d.1.1. Wenn Ja, wann war die ED? Datum:

- Wenn Nein oder unbekannt, wie wurde diagnostiziert bzw. ausgeschlossen?

5.d.1.2. Wurde eine Anamnese erhoben?

$$
\text { Ja o Nein o unbekannt } \theta
$$

5.d.1.2.1. Wenn Ja, wann? Datum:

5.d.1.3. Wurde der Nikotinabusus quantifiziert? Ja o Nein o

5.d.1.3.1. Wenn Ja, wann? Datum:

5.d.1.3.2. Wieviel und was wird geraucht?

5.d.1.3.3. $\quad \rightarrow \quad$ d.h.: Zigaretten in packyears:

5.d.2. Wenn bei der/dem Patientin/Patienten ein Nikotinabusus vorliegt; wurde der/dem Patientin/Patienten die Empfehlung gegeben das Rauchen zu stoppen? Ja o Nein o unbekannt $\theta$ 


\section{Hypertonie}

\section{Qualitätsindikatoren (QI)-Dokumentationsbogen zu Hypertonie-Screening, - Diagnostik und -Therapie}

Die zur Dokumentation benötigten Daten werden aus den Patientenakten entnommen. Der Dokumentationszeitraum ist: Beginn der jeweiligen hausärztlichen Betreuung bis heute.

H_1. Wann begann die hausärztliche Betreuung zwischen aktuellem Hausarzt und Patient? Datum:

H_2. Ist bei der Patientin oder dem Patienten eine Hypertonie diagnostiziert? Ja o Nein o

Wenn Ja $\quad \rightarrow \quad$ Bitte Datum-RR-Werte Tabelle ausfüllen (s.u.), dann weiter mit Diagnose

Wenn Nein $\quad \rightarrow \quad$ Bitte Datum-RR-Werte Tabelle ausfüllen (s.u.) \& anschliessend mit KHK und cholesterinsenkender Therapie weitermachen (Seite 20)

\section{Tabelle 3: Datum - RR-Wert - Tabelle}

In diese Tabelle werden alle in der Patientenakte dokumentierten RRWerte, Anthypertensiva und deren Dosierung eingetragen.

Tabelle bitte ggf. ergänzen

\begin{tabular}{|l|l|l|l|}
\hline Datum & RR-Wert $(\mathrm{mmHg})$ & Medikament & Dosierung \\
\hline & & & \\
& & & \\
& & & \\
& & & \\
\hline
\end{tabular}




$$
\mathbb{T}
$$




\section{Diagnose:}

\section{H_3. Medikation}

H_3.a.Wann wurde die Medikation mit Antihypertensiva begonnen?

Datum:

H_3.b. Mit welchem Medikament wurde die Therapie begonnen?

H_3.c. Mit welcher Dosierung wurde die Therapie begonnen?

\section{H_4. Patientendokumentation}

H_4.a.Führt die Praxis papierene Patientenakten?

Ja o Nein o

H_4.b.Führt die Praxis elektronische Patientenakten?

Ja o Nein o

H_4.c. Wo ist die Diagnose dokumentiert? Auf dem "'Titelblatt" oder der erste

Seite der papierenen Patientenakte?

Ja o Nein o

4.c.1. Falls Nein, wo ist die Diagnose dokumentiert? Bitte Ort angeben.

\begin{tabular}{|c|c|c|}
\hline gekennzeichnet? & Ja o & Nein o \\
\hline _4.e. Ist die Diagnose in der elektronischen Patientenakte & Daue & rdiagnose \\
\hline gekennzeichnet? & Ja o & Nein o \\
\hline H_4.f. Wird das Datum der ED (Erstdiagnose) angegeben? & Ja o & Nein o \\
\hline
\end{tabular}

\section{H_5. Erkrankungen und Risikofaktoren}

Zur Beantwortung der folgenden Items ist es wichtig, überall in der papierenen und elektronischen Patientenakte nach möglichen Erwähnungen der entsprechenden Pathologie zu fahnden. Z.B. auch ggf. vorliegende Patientenfragebögen nutzen

\section{H_5.a.PAVK in der Eigenanamnese}

\section{5.a.1. Ist die Diagnose pAVK dokumentiert? Ja o Nein o}

5.a.1.1. Wenn Ja, wann war die ED?

Datum:

- Wenn Nein, wie wurde überprüft, diagnostiziert bzw. ausgeschlossen? 
5.a.1.2. Wurde eine Anamnese erhoben? Ja o Nein o keine Angabe möglich $\theta$

5.a.1.2.1. Wenn Ja, wann? Datum:

5.a.1.3. Wurde ein Klinischer Befund erhoben? Ja o Nein o keine Angabe möglich $\theta$

5.a.1.3.1. Wenn Ja, wann? Datum:

5.a.1.4. Wurde weiterführende Diagnostik durchgeführt Ja o Nein o keine Angabe möglich $\theta$

- Wenn Ja, welche Diagnostik?

$\begin{array}{lll}\text { 5.a.1.4.1. } & \text { Peripherer Doppler } & \text { Ja o Nein o } \\ & \text { Datum: } & \\ \text { 5.a.1.4.2. } & \text { DSA } & \text { Ja o Nein o } \\ & \text { Datum: }\end{array}$

5.a.1.4.3. Sonstige Diagnostik Ja o Nein o

Datum:_ Welche Diagnostik?

\section{H_5.b. Diabetes}

5.b.1. Ist die Diagnose bereits bekannt? Ja o Nein o

5.b.1.1. Wenn Ja, wann war die ED? Datum

- Wenn Nein, wie wurde diagnostiziert, überprüft bzw. ausgeschlossen?)

5.b.1.2. Wurde eine Anamnese erhoben?

Ja o Nein o keine Angabe möglich $\theta$

5.b.1.2.1. Wenn Ja, wann? Datum:

5.b.1.3. Wurde ein Klinischer Befund erhoben?

Ja o Nein o keine Angabe möglich $\theta$

5.b.1.3.1. Wenn Ja, wann? Datum:

5.b.1.4. Wurde Labordiagnostik durchgeführt?

Ja o Nein o

- Wenn Ja, welche Diagnostik? 
5.b.1.4.1. Wurde der Nüchtern-BZ bestimmt? Ja o Nein o 5.b.1.4.1.1. Wenn Ja, wann? Datum Wert? $\mathrm{mg} / \mathrm{dl}$

5.b.1.4.2. Wurde ein OGTT durchgeführt? Ja o Nein o 5.b.1.4.2.1. Wenn Ja, wann? Datum Werte?

\section{H_5.c.Hyperlipidämie}

5.c.1. Ist die Diagnose bereits bekannt? Ja o Nein o

5.c.1.1. Wenn Ja, wann war die ED? Datum

- Wenn Nein, wie wurde diagnostiziert bzw. ausgeschlossen?

5.c.1.2. Wurde eine Anamnese erhoben?

$$
\text { Ja o Nein o keine Angabe möglich } \theta
$$

5.c.1.2.1. Wenn Ja, wann? Datum:

5.c.1.3. Wurde ein Klinischer Befund erhoben? Ja o Nein o keine Angabe möglich $\theta$

5.c.1.3.1. Wenn Ja, wann? Datum:

5.c.1.4. Wurde Labordiagnostik durchgeführt? Ja o Nein o

- Wenn Ja, welche Diagnostik?

5.c.1.4.1. Wurde das Gesamtcholesterin bestimmt? Ja o Nein o

5.c.1.4.2. Wurde HDL-/LDL-Cholesterin bestimmt? Ja o Nein o

5.c.1.4.3. Wenn Gesamtcholesterin und/oder HDL-/LDL-Cholesterin bestimmt wurde, bitte alle Werte mit dem jeweils entsprechenden Datum in die Cholesterin-Datum-Tabelle eintragen. (Seite 21) 


\section{H_5.e.Schädlicher Alkoholkonsum/Alkoholabhängigkeit}

5.e.1. Ist ein schädlicher Alkoholkonsum oder Alkoholabhängigkeit bereits bekannt? Ja o Nein o keine Angabe möglich $\theta$

5.e.1.1. Wenn Ja, was? Schädlicher Alkoholkonsum o Alkoholabhängigkeit $\quad \mathrm{o}$

5.e.1.2. Wann war die ED? Datum:

- Wenn Nein, wie wurde diagnostiziert bzw. ausgeschlossen?

5.e.1.3. Wurde eine Anamnese erhoben?

Ja o Nein o unbekannt $\theta$

5.e.1.3.1. Wenn Ja, wann? Datum

5.e.1.4. Wurde der Alkoholkonsum quantifiziert? Ja o Nein o Datum:

5.e.1.4.1. Wenn Ja, bitte Menge und Art des Alkohols/Tag angeben:

H_9.f.1. Wenn ein schädlicher Alkoholkonsum oder Alkoholabhängigkeit vorliegt, wurde der/dem Patientin/Patienten eine Begrenzung des Alkoholkonsums empfohlen?

Ja o Nein o

H_9.f.1.1. Wenn Ja, Wann?

H_6. (Labor-) tests

Wurden folgende Labortests oder Befunderhebungen durchgeführt?

H_6.a.Urinstix auf Protein Ja o Nein o

6.a.1. Wenn Ja, bitte alle Urinstix auf Protein-Ergebnisse mit Datum in u.a. (Labor-) Wertetabelle eintragen.

\section{H_6.b.Serumkreatinin Ja o Nein o}

6.b.1. Wenn Ja, bitte alle Serumkreatininwerte mit Datum in u.a. (Labor-) Wertetabelle eintragen. 
H_6.c. Natrium:

Ja o Nein o

6.c.1. Wenn Ja, bitte alle Serumnatriumwerte mit Datum in u.a. (Labor-)

Wertetabelle eintragen

H_6.d.Kalium:

Ja o Nein o

6.d.1. Wenn Ja, bitte alle Serumkaliumwerte mit Datum in u.a. (Labor-) Wertetabelle eintragen

H_6.e.EKG Ja o Nein o

6.e.1. Wenn Ja, bitte alle EKG-Befunde mit Datum in u.a. (Labor-)

Wertetabelle eintragen 
Tabelle 4: (Labor-) Wertetabelle zu den Punkten 6.a. bis 6.e.

In diese Tabelle werden alle in der elektronischen und/oder papierenen Patientenakte dokumentierten Laborergebnisse und EKG-Befunde eingetragen.

Bitte Einheiten beachten!

Tabelle bitte ggf. ergänzen

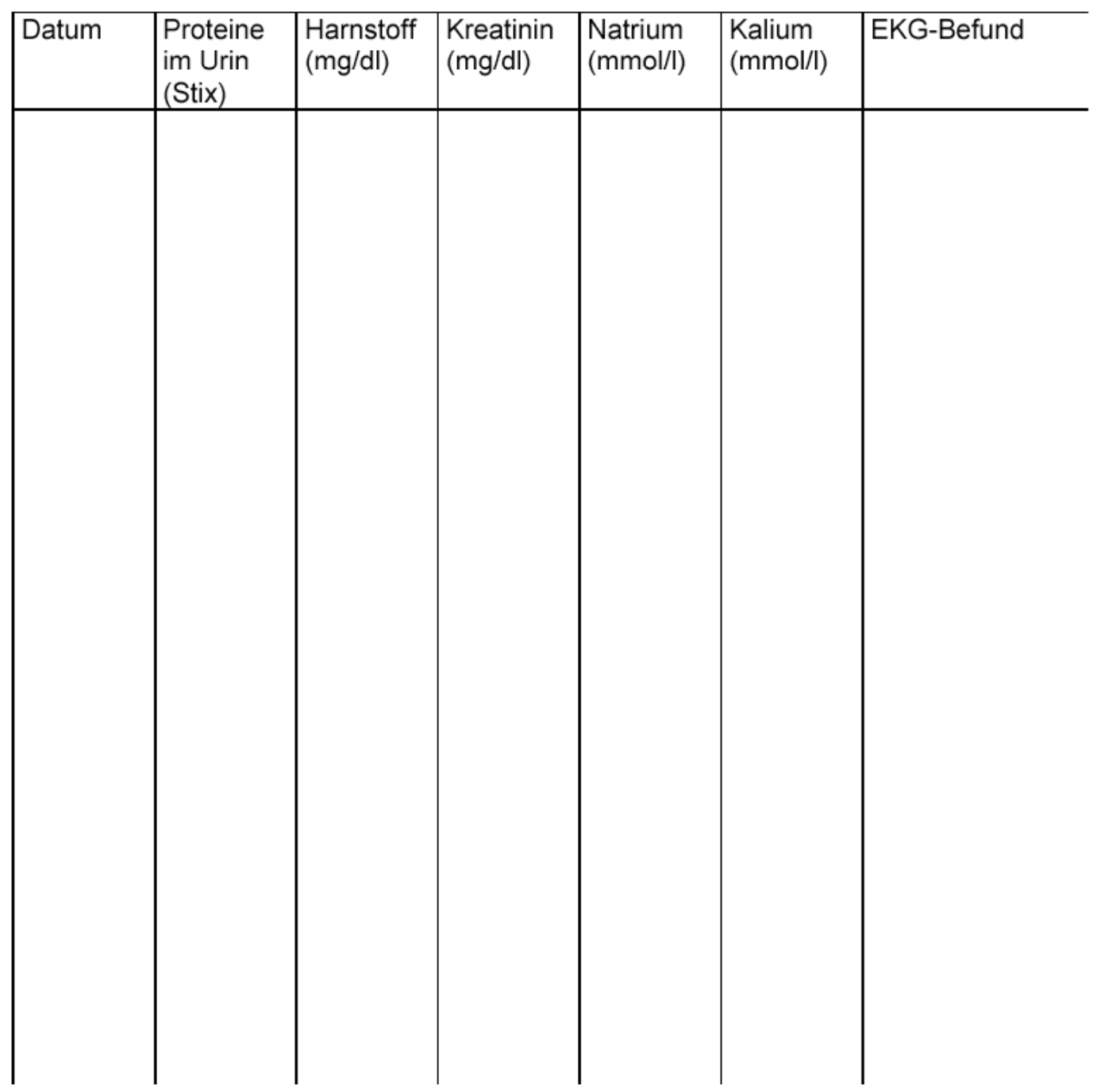


Therapie:

\section{H_7. Medikamentöse Behandlung?}

H_7.a.Hat der/die Patient/Patientin anhaltende (an mehr als 3 Messzeitpunkten) systolische RR-Werte von $\geq 160 \mathrm{mmHg}$ und/oder anhaltendem diastolische RR-Werte von $\geq 100 \mathrm{mmHg}$ ? (Siehe hierzu Datum - RR-Wert - Tabelle)

Ja o Nein o

Wenn $\mathrm{Ja}$, weiter mit $\mathrm{H} \_7 . b$. , wenn Nein, weiter mit Item $\mathrm{H} \_8$.

H_7.b. Wurden der/dem Patientin/Patienten nicht-medikamentöser Massnahmen zur RR-Senkung empfohlen?

Ja o Nein o unbekannt $\theta$

7.b.1. Wenn Ja, welche nicht-medikamentöser Massnahmen zur RR-Senkung wurden empfohlen?

Wann?

H_7.c. Wurde der/dem Patientin/Patienten eine medikamentöse Behandlung angeboten?

Ja o Nein o unbekannt $\theta$

7.c.1. Wenn Ja, Wann?

Welches Medikament?

Welche Dosierung?

7.c.2. Wenn Nein, Warum?

Aufgrund von Kontraindikationen (KIs)? Ja o Nein o unbek. $\theta$

7.c.2.1. Wenn Ja, welche KIs?

7.c.3. Wenn Nein, Warum?

Aufgrund von Intoleranzen?

Ja o Nein o unbek. $\theta$

7.c.3.1. Wenn Ja, welche Intoleranzen?

7.c.4. Wenn Nein, Warum?

Aufgrund von sonstigen Gründen? Ja o Nein o 
7.c.4.1. Wenn Ja, welche sonstigen Gründe?

\section{H_8. Medikamentöse Behandlung bei Komorbidität?}

H_8.a.Hat der/die Patient/Patientin anhaltende (an mehr als 3 Messzeitpunkten) systolische RR-Werte von 140-159 mmHg und/oder anhaltende diastolische RR-Werte von 90-99 mmHg? (Siehe hierzu Datum - RR-Wert - Tabelle)

Ja o Nein o

Wenn Ja, weiter mit $\mathrm{H} \_8 . b$., wenn Nein, weiter mit Item $\mathrm{H} \_9$.

H_8.b. Wurden der/dem Patientin/Patienten nicht-medikamentöser Massnahmen zur RR-Senkung empfohlen?

Ja o Nein o unbekannt $\theta$

8.b.1. Wenn Ja, welche nicht-medikamentöser Massnahmen zur RR-Senkung wurden empfohlen?

\section{Wann?}

H_8.c. Wurde der/dem Patientin/Patienten eine medikamentöse Behandlung angeboten?

Ja o Nein o unbekannt $\theta$

8.c.1. Wenn Ja, Wann?

Welches Medikament?

Welche Dosierung?

- Wenn Nein, Warum?

8.c.2. Aufgrund von Kontraindikationen (KIs)? Ja o Nein o unbekannt $\theta$ 8.c.2.1. Wenn Ja, welche KIs?

\section{8.c.3. Aufgrund von Intoleranzen? Ja o Nein o unbekannt $\theta$}

8.c.3.1. Wenn Ja, welche Intoleranzen?

8.c.4. Aufgrund von sonstigen Gründen?

Ja o Nein o 
8.c.4.1. Wenn Ja, welche sonstigen Gründe?

\section{H_9. Nicht-medikamentöse Massnahmen?}

H_9.a.Wie ist der BMI der/des Patientin/Patienten?

BMI (Angaben in der Patientenakte) Datum:

H_9.b.Wie ist die Körpergröße in m: Datum:

H_9.c. Wie ist das Körpergewicht in kg: Datum:

H_9.d. $\rightarrow$ Errechneter BMI: kg KG/ Körpergröße in $\mathrm{m}^{2}$

H_9.e.Ist der BMI $>30$ ? Ja o Nein o

9.e.1. Wenn Ja, wurde der/dem Patientin/Patienten eine Gewichtsreduktion empfohlen?

Ja o Nein o unbekannt $\theta$

9.e.1.1. Wenn Ja, Wann?

\section{H_10. Aktuelle Medikation}

Mit welchen antihypertensiven Medikamenten wird der Patient aktuell behandelt?

H_10.a. $\quad \beta$-Blocker ja nein unbek.

H_10.b. Diuretikum

H_10.c. Ca-Antagonist

H_10.d. ACE-Hemmer

H_10.e. AT1-Antagonist

H_10.f. Nitropräparate

H_10.g. Alpha 1 Rezeptorantagonisten

H_10.h. Sonstige bitte dokumentieren:

\section{H_11. Schwangere?}
H_11.a. Ist die Pat. schwanger?
Ja o Nein o 


\section{H_12. Therapieänderung?}

H_12.a. Liegen bei der/dem Pat. unter antihypertensiver Therapie an 3 oder mehr Messzeitpunkten RR-Werte $>150 / 90$ mmHg vor?

Zur Beantwortung dieser Frage, siehe Daten der Datum - RR-Wert Tabelle.

Ja o Nein o

12.a.1. Wenn Ja, wird eine Therapieänderung angeboten?

Ja o Nein o unbekannt $\theta$

12.a.1.1. Wenn Ja, Wann? Datum

12.a.2. Wird ein neues Medikament angeboten? Ja o Nein o unbekannt $\theta$

12.a.2.1. Wenn Ja, welches Medikament wird angeboten?

12.a.2.2. Wenn Nein, wird die Dosierung verändert? Ja o Nein o unbekannt $\theta$

12.a.2.2.1. Wenn Ja, wie wird die Dosierung verändert? Von:

Nach:

12.a.2.2.2. Wenn Nein, warum? (Was ist dokumentiert?) 


\section{KHK und cholesterinsenkende Therapie}

Qualitätsindikatoren (QI)-Dokumentationsbogen zur Diagnostik der Komorbidität KHK und cholesterinsenkenden Therapie

Die zugrundeliegenden Daten werden aus den digitalen und handschriftlichen Patientenakten der Hausärztinnen und Hausärzte entnommen.

\section{K_1. Ist bei der Patientin oder dem Patienten eine KHK diagnostiziert?}

Ja o Nein o

Wenn Ja $\quad \rightarrow$ Weiter mit Diagnose

Wenn Nein $\rightarrow$ Dokumentation hier beenden

\section{Diagnose}

\section{K_2. Patientendokumentation?}

K_2.a.Wo ist die Diagnose dokumentiert? Auf dem "'Titelblatt" oder der erste Seite der papierenen Patientenakte?

Ja o Nein o

2.a.1. Falls Nein, wo ist die Diagnose dokumentiert? Bitte Ort angeben.

K_2.b. Ist die Diagnose in der papierenen Patientenakte als Dauerdiagnose gekennzeichnet?

Ja o Nein o

K_2.c. Ist die Diagnose in der elektronischen Patientenakte als Dauerdiagnose gekennzeichnet?

Ja o Nein o

K_2.d. Wird das Datum der ED (Erstdiagnose) angegeben? Ja o Nein o 2.d.1. Wenn Ja, wann war die ED? Datum: 


\section{Therapie}

K_3. Ist das Serumcholesterin innerhalb der letzten 5 Jahre gemessen worden? (Bedingt durch die Dokumentationsbogenstruktur können die Inhalte dieses Items bereits unter $5 \mathrm{c}$ abgefragt worden sein)

\section{Ja o Nein o}

K_3.a.Wenn Ja, wann? Datum: ___ Wert?

K_3.b. Wurde HDL-/LDL-Cholesterin bestimmt? Ja o Nein o

3.b.1. Wenn Ja, wann?

3.b.2. Werte? HDL LDL

K_3.c. Bei häufigeren Cholesterin-, HDL oder LDL-Messungen, bitte auf angehängtem Cholesterin - Datum - Tabelle eintragen.

K_4. Ist bei einem Gesamtcholesterin von $>200 \mathrm{mg} / \mathrm{dl}$ dem Patienten eine Ernährungsberatung oder eine medikamentöse lipidsenkende Therapie oder eine Therapieänderung (falls in den vorangegangenen 6 Monaten nicht geändert) angeboten worden?

K_4.a. Liegt oder lag das Gesamtcholesterin $>200 \mathrm{mg} / \mathrm{dl}$ ? $\quad$ Ja o Nein o

K_4.b. Wenn das Gesamtcholesterin $>200 \mathrm{mg} / \mathrm{dl}$ liegt oder lag,

4.b.1. wurde eine Ernährungsberatung angeboten? Ja o Nein o

4.b.1.1. wenn Ja, wann? Datum:

oder

4.b.2. wurde eine medikamentöse lipidsenkende Therapie angeboten? Ja o Nein o

4.b.2.1. wenn Ja, wann? Datum:

4.b.2.2. welches Medikament?

4.b.2.3. in welcher Dosierung?

oder

4.b.3. wurde eine Therapieänderung angeboten? Ja o Nein o

4.b.3.1. wenn Ja, wann? Datum:

4.b.3.2. Wurde eine Dosierung geändert? Ja o Nein o

4.b.3.2.1. wenn Ja, welches Medikament?

4.b.3.2.2. Dosierungsänderung von: nach: $\mathrm{mg}$ $\mathrm{mg}$,

4.b.3.3. Wurde ein Medikament neu angesetzt? Ja o Nein o

4.b.3.3.1. Wenn Ja, welches Medikament?

4.b.3.3.2. in welcher Dosierung?

4.b.3.4. Wurde ein Medikament abgesetzt? Ja o Nein o 
4.b.3.4.1. Wenn Ja, welches Medikament?

Tabelle 5: Cholesterin - Datum - Tabelle

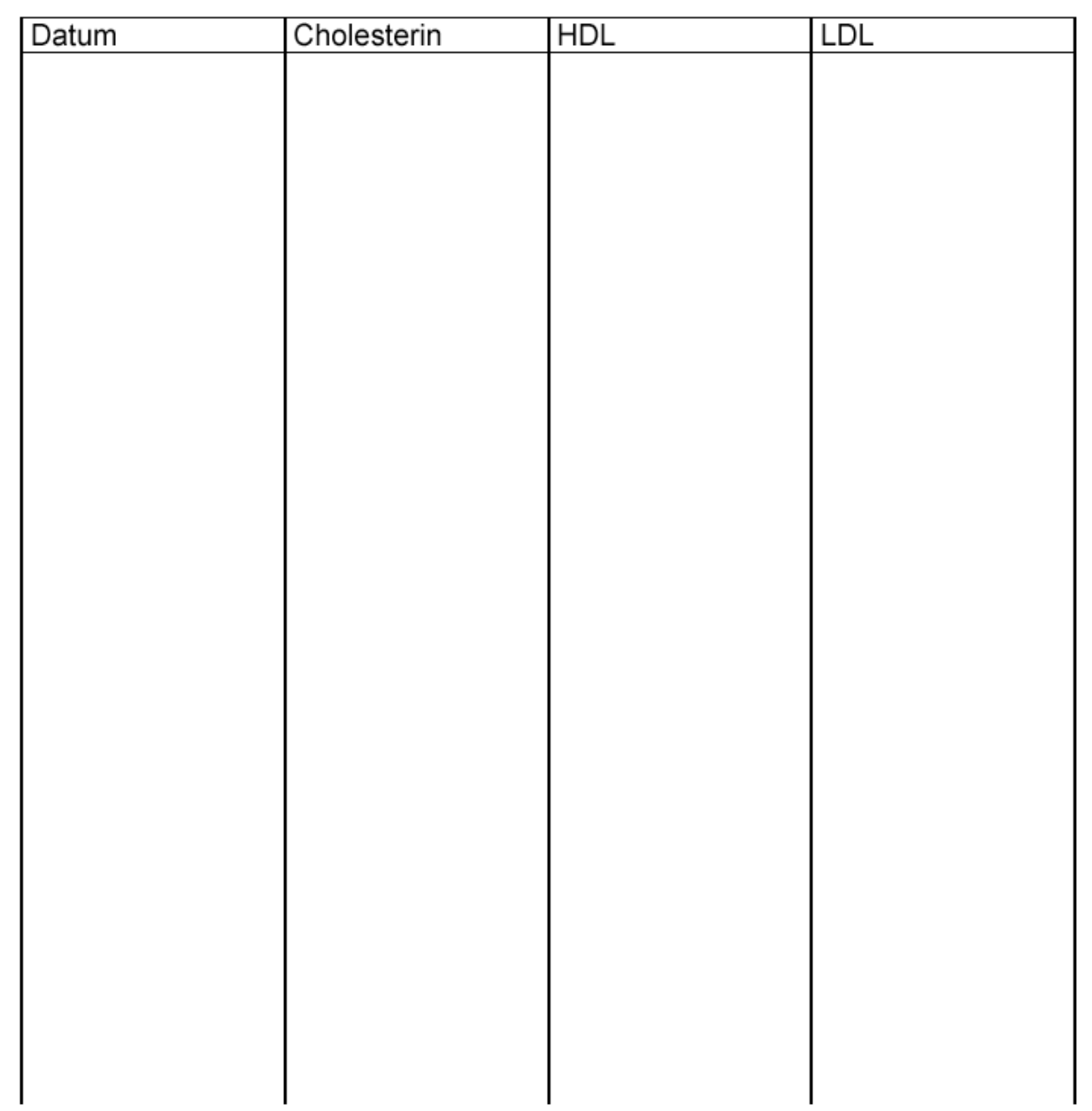

Vielen Herzlichen Dank für das Ausfüllen dieser Dokumentation ! 